\title{
COHOMOLOGY AND CONFORMAL DERIVATIONS OF BIHOM-LIE CONFORMAL SUPERALGEBRAS
}

\section{TAOUFIK CHTIOUI}

University of Sfax

Faculty of Sciences Sfax

BP 1171, 3038 Sfax

Tunisia

e-mail: chtioui.taoufik@yahoo.fr

\begin{abstract}
In this paper, we introduce the notion of BiHom-Lie conformal superalgebras. We develop its representation theory and define the cohomology group with coefficients in a module. Finally, we introduce conformal derivations of BiHomLie conformal superalgebras and study some of their properties.
\end{abstract}

2020 Mathematics Subject Classification: 30C35, 17B39, 14F25, 20G05, 13 N15.

Keywords and phrases: BiHom-Lie conformal superalgebra, representation, cohomology, conformal derivation.

Received November 27, 2020

(C) 2021 Scientific Advances Publishers

This work is licensed under the Creative Commons Attribution International License (CC BY 3.0).

http://creativecommons.org/licenses/by/3.0/deed.en_US

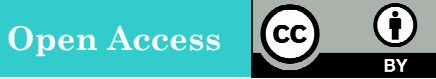




\section{Introduction}

The notion of Lie conformal (super)algebras are introduced by Kac in [10], in which he gave an axiomatic description of the singular part of the operator product expansion of chiral fields in conformal field theory. On the other hand, it is a useful tool to study vertex (super)algebras and has many applications in the theory of Lie superalgebras. Moreover, it is equivalent to the notion of a linear Hamiltonian operator introduced in [6], and in [14], Zhao et al. developed deformation of Lie conformal superalgebras and introduced derivations of Lie conformal superalgebras and study their properties.

In [1], authors introduced the notion of Hom-Lie superalgebras and they gave a classification of Hom-Lie admissible superalgebras. Later, Makhlouf et al. studied the representation and the cohomology of HomLie superalgebras in [2] and calculated the derivations and the second cohomology group of $q$-deformed Witt superalgebra. In [13], Yuan introduced the notion of Hom-Lie conformal superalgebra and proved that a Hom-Lie conformal superalgebra is equivalent to a Hom-Gel'fandDorfman super bialgebra. In [15], the authors developed cohomology theory of Hom-Lie conformal algebras, discussed some applications to the study of deformations of regular Hom-Lie conformal algebras and introduced derivations of multiplicative Hom-Lie conformal algebras. Motivated by these results, authors, in [8], introduced the notion of representation theory of Hom-Lie conformal superalgebras and discuss the cases of adjoint representations. Furthermore, they developed their cohomology group and discuss some applications to the study of deformation theory.

Motivated by a categorical study of Hom-algebra and new type of categories, the authors introduced, in [5], a generalized algebraic structure dealing with two commuting multiplicative linear maps, called BiHom-algebras including BiHom-associative algebras and BiHom-Lie algebras. Recently, Zhao, Yuan and Chen developed the cohomology and deformation theory of BiHom-Lie conformal algebras and investigated the notion of conformal derivations of BiHom-Lie conformal algebras in [9]. 
The main goal of the present work is to introduce the notion of BiHom-Lie conformal superalgebras, study their cohomology theory and investigate the notion of generalized conformal derivations.

The paper is organized as follows. In Section 1, we recall some basic definitions and results about the notion of Lie conformal (super)algebras. In Section 2, we introduce the notion BiHom-Lie conformal superalgebras illustrated by some examples give some related results. In Section 3, we develop representation and cohomology theory of BiHom-Lie conformal superalgebras. Section 4 is devoted to the study of derivations and generalized derivations of BiHom-Lie conformal superalgebras and their properties.

\section{Preliminaries}

Throughout the paper, all algebraic systems are supposed to be over a field $\mathbb{C}$, of characteristic 0 and denote by $\mathbb{Z}_{+}$the set of all nonnegative integers and by $\mathbb{Z}$ the set of all integers.

Let $V$ be a superspace that is a $\mathbb{Z}_{2}$-graded linear space with a direct sum $V=V_{0} \oplus V_{1}$. The elements of $V_{j}, j=[0,1]$, are said to be homogeneous and of parity $j$. The parity of a homogeneous element $x$ is denoted by $|x|$. Throughout what follows, if $|x|$ occurs in an expression, then it is assumed that $x$ is homogeneous and that the expression extends to the other elements by linearity.

Definition 1.1. A Lie conformal superalgebra $\mathcal{R}$ is a left $\mathbb{Z}_{2}$-graded $\mathbb{C}[\partial]$-module, and for any $n \in \mathbb{Z}_{\geq 0}$ there is a family of $\mathbb{C}$-linear $n$-products from $\mathcal{R} \otimes \mathcal{R}$ to $\mathcal{R}$ satisfying the following conditions:

(C0) For any $a, b \in \mathcal{R}$, there is an $N$ such that $a_{(n)} b=0$ for $n \gg N$;

(C1) For any $a, b \in \mathcal{R}$ and $n \in \mathbb{Z}_{\geq 0},(\partial a)_{(n)} b=-n a_{(n)} b$;

(C2) For any $a, b \in \mathcal{R}$ and $n \in \mathbb{Z}_{\geq 0}$,

$$
a_{(n)} b=-(-1)^{|a||b|} \sum_{j=0}^{\infty}(-1)^{j+n} \frac{1}{j !} \partial^{j}\left(b_{(n+j)} a\right)
$$


(C3) For any $a, b, c \in \mathcal{R}$ and $m, n \in \mathbb{Z}_{\geq 0}$,

$$
a_{(m)}\left(b_{(n)} c\right)=\sum_{j=0}^{m}\left(\begin{array}{c}
m \\
j
\end{array}\right)\left(a_{(j)} b\right)_{(m+n-j)} c+(-1)^{|a||b|} b_{(n)}\left(a_{(m)} c\right)
$$

(Convention: $a_{(n)} b=0$ if $n<0$ ). Note that if we define $\lambda$-bracket $[-\lambda-]:$

$$
\left[a_{\lambda} b\right]=\sum_{n=0}^{\infty} \frac{\lambda^{n}}{n !} a_{(n)} b, a, b \in \mathcal{R}
$$

That is, $\mathcal{R}$ is a Lie conformal superalgebra if and only if $\left[-\lambda^{-}\right]$ satisfies the following axioms:

$(\mathrm{C} 0)_{\lambda}\left[\mathcal{R}_{i \lambda} \mathcal{R}_{j}\right] \subseteq \mathcal{R}_{i+j}[\lambda], \forall i, j \in \mathbb{Z}_{2} ;$

$(\mathrm{C} 1)_{\lambda}$ Conformal sesquilinearity: $\left[(\partial a)_{\lambda} b\right]=-\lambda\left[a_{\lambda} b\right]$

$(\mathrm{C} 2)_{\lambda}$ Skew-symmetry: $\left[a_{\lambda} b\right]=-(-1)^{|a||b|}\left[b_{-\lambda-\lambda} a\right]$

(C3) $)_{\lambda}$ Jacobi identity: $\left[a_{\lambda}\left[b_{\mu} c\right]\right]=\left[\left[a_{\lambda} b\right]_{\lambda+\mu} c\right]+(-1)^{|a||b|}\left[b_{\mu}\left[a_{\lambda} c\right]\right]$.

Similarly, an associative conformal superalgebra is a left $\mathbb{Z}_{2}$-graded $\mathbb{C}[\partial]$-module $\mathcal{R}$ equipped with a $\mathbb{C}$-linear map $\cdot_{\lambda}: \mathcal{R} \otimes \mathcal{R} \rightarrow \mathcal{R}[\lambda]$ such that $\mathcal{R}_{i \lambda} R_{j} \subseteq \mathcal{R}_{i+j}[\lambda]$ and

$(\mathrm{C} 1)_{\lambda}$ Conformal sesquilinearity: $\left[(\partial a)_{\lambda} b\right]=-\lambda\left[a_{\lambda} b\right]$

(C2) $)_{\lambda}^{\prime}$ Associativity: $\left(a_{\lambda} b\right)_{\lambda+\mu} c=a_{\lambda}\left(b_{\mu} c\right)$.

$\mathcal{R}$ is called finite if it is finitely generated as a $\mathbb{C}[\partial]$-module. 
Proposition 1.1. Let $(\mathcal{R}, \cdot \lambda)$ be an associative conformal superalgebra. Then $\left(\mathcal{R},\left[\cdot_{\lambda} \cdot\right]\right)$ is a Lie conformal superalgebra, where for any homogeneous elements $a, b \in \mathcal{R}$

$$
\left[a_{\lambda} b\right]=a_{\lambda} b-(-1)^{|a||b|} b_{-\lambda-\lambda} a
$$

Example 1.1. Let $\mathcal{R}$ be a Lie conformal superalgebra and $B$ be a commutative associative (ordinary) superalgebra. Then $\mathcal{R} \otimes B$ carries a Lie conformal superalgebra structure defined as follows. The $\mathbb{C}[\partial]$ module structure is given by $\partial(r \otimes b)=(\partial r) \otimes b(r \in \mathcal{R}, b \in B)$, and the $\lambda$-bracket by

$$
\left[(r \otimes b)_{\lambda}\left(r^{\prime} \otimes b^{\prime}\right)\right]=(-1)^{|b|\left|r^{\prime}\right|}\left[r_{\lambda} r^{\prime}\right] \otimes\left(b b^{\prime}\right)
$$

Notice that if $\mathcal{R}$ is finite and $B$ is finite-dimensional, then $\mathcal{R} \otimes B$ is also finite.

A first generalization of Lie conformal superalgebras is the notion of Hom-Lie conformal superalgebras introduced in [12].

Definition 1.2. A Hom-Lie conformal superalgebra $R=R_{\overline{0}} \oplus R_{\overline{1}}$ is a $\mathbb{Z}_{2}$-graded $\mathbb{C}[\partial]$-module equipped with an even linear endomorphism $\alpha$ such that $\alpha \partial=\partial \alpha$, and a $\mathbb{C}$-linear map

$$
R \otimes R \rightarrow \mathbb{C}[\lambda] \otimes R, \quad a \otimes b \mapsto\left[a_{\lambda} b\right]
$$

such that $\left[R_{i \lambda} R_{j}\right] \subseteq R_{i+j i}[\lambda], i, j \in \mathbb{Z}_{2}$, and the following axioms hold for $a, b, c \in R$ :

$$
\begin{aligned}
& {\left[\partial a_{\lambda} b\right]=-\lambda\left[a_{\lambda} b\right],\left[a_{\lambda} \partial b\right]=(\partial+\lambda)\left[a_{\lambda} b\right]} \\
& {\left[a_{\lambda} b\right]=-(-1)^{|a||b|}\left[b_{-\lambda-\partial} a\right],} \\
& {\left[\alpha(a)_{\lambda}\left[b_{\mu} c\right]\right]=\left[\left[a_{\lambda} b\right]_{\lambda+\mu} \alpha(c)\right]+(-1)^{|\alpha||b|}\left[\alpha(b)_{\mu}\left[a_{\lambda} c\right]\right] .}
\end{aligned}
$$


Definition 1.3. A BiHom-Lie conformal algebra is a BiHom-conformal algebra $(A,[\cdot \lambda \cdot], \alpha, \beta)$ such that the following axioms hold for any $a, b, c \in A$ :

$$
\begin{aligned}
& \alpha\left(\left[a_{\lambda} b\right]\right)=\left[\alpha(a)_{\lambda} \alpha(b)\right], \beta\left(\left[a_{\lambda} b\right]\right)=\left[\beta(a)_{\lambda} \beta(b)\right], \\
& {\left[\beta(a)_{\lambda} \alpha(b)\right]=-\left[\beta(b)_{-\lambda-\lambda} \alpha(a)\right],} \\
& {\left[\alpha \beta(a)_{\lambda}\left[b_{\mu} c\right]\right]=\left[\left[\beta(a)_{\lambda} b\right]_{\lambda+\mu} \beta(c)\right]+\left[\beta(b)_{\mu}\left[\alpha(a)_{\lambda} c\right]\right] .}
\end{aligned}
$$

In particular, if $\alpha, \beta$ are algebra isomorphisms, then $(A,[\cdot \lambda \cdot], \alpha, \beta)$ is called regular.

Definition 1.4. A module $(V, \phi, \psi)$ over a BiHom-Lie conformal algebra $\left(A\left[\lambda_{\lambda}\right], \alpha, \beta\right)$ is a $\mathbb{C}[\partial]$-module endowed with two commuting $\mathbb{C}$-linear maps $\phi, \psi$ and a $\mathbb{C}$-bilinear map $A \otimes V \rightarrow V[\lambda], a \otimes v \mapsto a_{\lambda} v$, such that for $a, b \in A, v \in V$ :

$$
\begin{gathered}
\psi \circ \partial=\partial \circ \psi, \phi \circ \partial=\partial \circ \phi, \\
(\partial a)_{\lambda} v=-\lambda\left(a_{\lambda} v\right), a_{\lambda}(\partial v)=(\partial+\lambda) a_{\lambda} v, \\
\phi\left(a_{\lambda} v\right)=\alpha(a)_{\lambda}(\phi(\psi)), \psi\left(a_{\lambda} v\right)=\beta(a)_{\lambda}(\psi(v)), \\
{\left[\beta(a)_{\lambda} b\right]_{\lambda+\mu} \psi(v)=\alpha \beta(a)_{\lambda}\left(b_{\mu} v\right)-\beta(b)_{\mu}\left(\alpha(a)_{\lambda} v\right) .}
\end{gathered}
$$

Remark 1.1. By Definition 1.4, it is easy to see hat a module over a BiHom-Lie conformal algebra $A$ in a finite $\mathbb{C}[\partial]$-module $V$ is the same as a homomorphism of BiHom-Lie conformal algebra $\rho: A \rightarrow g c(V)$ which is called a representation of $A$. The identity (1.11) can be written as

$$
\rho\left(\left[\beta(a)_{\lambda} b\right]\right)_{\lambda+\mu} \circ \psi=\rho(\alpha \beta(a))_{\lambda} \circ \rho(b)_{\mu}-\rho(\beta(b))_{\mu} \circ \rho(\alpha(a))_{\lambda},
$$

for all $a, b \in A$. In addition, $\rho$ satisfy $\rho(\partial a)_{\lambda} v=-\lambda \rho(a)_{\lambda} v, \forall a \in A, v \in V$.

The adjoint representation of $A$ is denoted by $a d$, i.e., $\operatorname{ad}(a)_{\lambda}(b)=$ $\left[a_{\lambda} b\right]$, where $a, b \in A$. 
Definition 1.5. A BiHom-associative conformal superalgebra $R$ is a $\mathbb{Z}_{2}$-graded $\mathbb{C}[\partial]$-module equipped with two even linear endomorphisms $\alpha, \beta$ and endowed with a $\lambda$-product from $\mathcal{R} \otimes \mathcal{R}$ to $\mathbb{C}[\partial] \otimes \mathcal{R}$, for any $a, b, c \in \mathcal{R}$, satisfying the following conditions:

$$
\begin{gathered}
(\partial a)_{\lambda} b=-\lambda a_{\lambda} b, a_{\lambda}(\partial b)=(\partial+\lambda) a_{\lambda} b, \\
\alpha \partial=\partial \alpha, \beta \partial=\partial \beta \\
\alpha\left(a_{\lambda} b\right)=\alpha(a)_{\lambda} \alpha(b), \beta\left(a_{\lambda} b\right)=\beta(a)_{\lambda} \beta(b), \\
\alpha(a)_{\lambda}\left(b_{\mu} c\right)=\left(a_{\lambda} b\right)_{\lambda+\mu} \alpha(c) .
\end{gathered}
$$

Definition 1.6. A subset $U$ of a BiHom-conformal (super)-algebra $(A, \lambda, \alpha, \beta)$ is called a BiHom-conformal subalgebra if $\alpha(U) \subseteq A$, $\beta(U) \subseteq A$ and $a_{\lambda} b \in U$, for any $a, b \in U$.

A subset $U A$ is called a left BiHom-conformal ideal (resp., right BiHom-conformal ideal) if $\alpha(U) \subseteq A, \beta(U) \subseteq A$ and $a_{\lambda} b \in U$, for any $a \in A, b \in U$. (resp., for any $a \in U, b \in A$ ).

\section{Definitions and Examples}

In this section, we introduce the notion of BiHom-Lie conformal superalgebra, which is a BiHom-generalization of (Hom)-Lie conformal superalgebras and also a superanalogue of (Bi)Hom-Lie conformal algebras.

Definition 2.1. A BiHom-Lie conformal superalgebra $\mathcal{R}=\mathcal{R}_{0} \oplus \mathcal{R}_{1}$ is a $\mathbb{Z}_{2}$-graded $\mathbb{C}[\partial]$-module equipped with two commuting linear maps $\alpha, \beta$ and a $\lambda$-bracket $[\cdot \lambda \cdot]$ which defines a $\mathbb{C}$-linear map from $\mathcal{R} \otimes \mathcal{R}$ to $R[\lambda]=\mathbb{C}[\lambda] \otimes \mathcal{R}$ such that $\left[\mathcal{R}_{i \lambda} \mathcal{R}_{j}\right] \subseteq R_{i+j}[\lambda], i, j \in \mathbb{Z}_{2}$ and the following axioms hold for all homogeneous elements $a, b, c \in \mathcal{R}$ : 


$$
\begin{gathered}
\alpha \partial=\partial \alpha, \beta \partial=\partial \beta, \\
\alpha\left(\left[a_{\lambda} b\right]\right)=\left[\alpha(a)_{\lambda} \alpha(b)\right], \beta\left(\left[a_{\lambda} b\right]\right)=\left[\beta(a)_{\lambda} \beta(b)\right], \\
{\left[\partial a_{\lambda} b\right]=-\lambda\left[a_{\lambda} b\right],\left[a_{\lambda} \partial b\right]=(\partial+\lambda)\left[a_{\lambda} b\right],} \\
{\left[\beta(a)_{\lambda} \alpha(b)\right]=-(-1)^{|a||b|}\left[\beta(b)_{-\lambda-\partial} \alpha(a)\right],} \\
{\left[\alpha \beta(a)_{\lambda}\left[b_{\mu} c\right]\right]=\left[\left[\beta(a)_{\lambda} b\right]_{\lambda+\mu} \beta(c)\right]+(-1)^{|a||b|}\left[\beta(b)_{\mu}\left[\alpha(a)_{\lambda} c\right]\right] .}
\end{gathered}
$$

A BiHom-Lie conformal superalgebra $(\mathcal{R}, \alpha, \beta)$ is called finite if $\mathcal{R}$ is a finitely generated $\mathbb{C}[\partial]$-module. The rank of $\mathcal{R}$ is its rank as a $\mathbb{C}[\partial]$-module. If $\alpha$ and $\beta$ are algebra isomorphisms, then $(\mathcal{R}, \alpha, \beta)$ is called regular.

We recover Lie conformal superalgebras when $\alpha=\beta=i d$. The BiHom-Lie conformal algebras are obtained when the odd part is trivial.

A linear map $\rho: \mathcal{R} \rightarrow \mathcal{R}^{\prime}$ is a homomorphism of BiHom-Lie conformal superalgebras if $\rho$ satisfies $\rho \partial=\partial \rho, \rho \alpha=\alpha^{\prime} \rho, \rho \beta=\beta^{\prime} \rho$ and $\rho\left(\left[a_{\lambda} b\right]\right)=\left[\rho(a)_{\lambda} \rho(b)\right]$, for all $a, b \in \mathcal{R}$.

Example 2.1. Let $g=g_{0} \oplus g_{1}$ be a BiHom-Lie superalgebra with Lie bracket $[-,-]$ and with structure maps $\alpha$ and $\beta$. Let $(\mathrm{Curg})_{\theta}:=\mathbb{C}[\partial]$ $\otimes g_{\theta}$ be the free $\mathbb{C}[\partial]$-module. Then $\operatorname{Curg}=(\mathrm{Curg})_{0} \oplus(\mathrm{Curg})_{1}$ is a BiHom-Lie conformal superalgebra with $\lambda$-bracket given by

$$
\begin{aligned}
& \alpha(f(\partial) \otimes a)=f(\partial) \otimes \alpha(a), \\
& \beta(f(\partial) \otimes a)=f(\partial) \otimes \beta(a), \\
& {\left[(f(\partial) \otimes a)_{\lambda}(g(\partial) \otimes b)\right]=f(-\lambda) g(\partial+\lambda) \otimes[a, b], \forall a, b \in g .}
\end{aligned}
$$


Example 2.2. Let $(L,[\cdot, \cdot], \alpha, \beta)$ be a regular BiHom-Lie superalgebra. Denote by $\hat{L}=L \otimes \mathbb{C}\left[t, t^{-1}\right]$ the affization of $L$ with

$$
\left[u \otimes t^{m}, v \otimes t^{n}\right]=[u, v] \otimes t^{m+n},\left|u \otimes t^{n}\right|=|u|,
$$

for any $u, v \in L, m, n \in \mathbb{Z}$. Extend $\alpha, \beta$ to $\hat{L}$ by $\alpha\left(u \otimes t^{m}\right)=\alpha(u) \otimes t^{m}$ and $\beta\left(u \otimes t^{m}\right)=\beta(u) \otimes t^{m}$. Then $(\hat{L},[\cdot],, \alpha, \beta)$ is a BiHom-Lie superalgebra. By simple verification, we get a BiHom-Lie conformal superalgebra $\mathcal{R}=\mathbb{C}[\partial] L$ with

$$
\left[u_{\lambda} v\right]=[u, v], \alpha(f(\partial) u)=f(\partial) \alpha(u), \beta(f(\partial) u)=f(\partial) \beta(u),
$$

for any $u, v \in L$.

Example 2.3. Let $\mathcal{R}=\mathbb{C}[\partial] L \oplus \mathbb{C}[\partial] E$ be a free $\mathbb{Z}_{2}$-graded $\mathbb{C}[\partial]$-module. Define

$$
\begin{aligned}
& \alpha(L)=f(\partial) L, \alpha(E)=g(\partial) E, \\
& \beta(L)=L, \beta(E)=E, \\
& {\left[L_{\lambda} L\right]=(\partial+2 \lambda) L,\left[L_{\lambda} E\right]=\left(\partial+\frac{3}{2} \lambda\right) E,} \\
& {\left[E_{\lambda} L\right]=\left(\frac{1}{2} \partial+\frac{3}{2} \lambda\right) E,\left[E_{\lambda} E\right]=0 .}
\end{aligned}
$$

Then $(\mathcal{R}, \alpha, \beta)$ is a BiHom-Lie conformal superalgebra, where $R_{0}=\mathbb{C}[\partial] L$ and $R_{1}=\mathbb{C}[\partial] E$.

Example 2.4. Let $\mathcal{R}=\mathbb{C}[\partial] e_{1} \oplus \mathbb{C}[\partial]\left(e_{2}+e_{3}\right)$ be a free $\mathbb{Z}_{2}$-graded $\mathbb{C}[\partial]$-module and

$$
e_{1}=\left(\begin{array}{lll}
0 & 0 & 1 \\
0 & 0 & 0 \\
0 & 0 & 0
\end{array}\right), e_{2}=\left(\begin{array}{lll}
0 & 1 & 0 \\
0 & 0 & 0 \\
0 & 0 & 0
\end{array}\right), e_{3}=\left(\begin{array}{lll}
0 & 0 & 0 \\
0 & 0 & 1 \\
0 & 0 & 0
\end{array}\right) .
$$


Define

$$
\begin{aligned}
& \alpha\left(e_{1}\right)=e_{1}, \alpha\left(e_{2}\right)=e_{3}, \alpha\left(e_{3}\right)=e_{2}, \\
& \beta\left(e_{1}\right)=e_{1}, \beta\left(e_{2}\right)=-e_{3}, \beta\left(e_{3}\right)=-e_{2}, \\
& {\left[e_{1 \lambda} e_{1}\right]=\left[e_{2 \lambda} e_{2}\right]=\left[e_{3 \lambda} e_{3}\right]=0,\left[e_{1 \lambda} e_{2}\right]=\left[e_{1 \lambda} e_{3}\right]=0,\left[e_{2 \lambda} e_{3}\right]=e_{1} .}
\end{aligned}
$$

One may check directly that $(\mathcal{R}, \alpha, \beta)$ is a BiHom-Lie conformal superalgebra.

Proposition 2.1. Let $\mathcal{R}$ be a Lie conformal superalgebra and $\alpha, \beta: \mathcal{R} \rightarrow \mathcal{R}$ two even commuting linear maps such that

$$
\alpha \partial=\partial \alpha, \beta \partial=\partial \beta, \alpha\left(\left[a_{\lambda} b\right]\right)=\left[\alpha(a)_{\lambda} \alpha(b)\right], \beta\left(\left[a_{\lambda} b\right]\right)=\left[\beta(a)_{\lambda} \beta(b)\right] .
$$

Define a $\mathbb{C}$-linear map from $\mathcal{R} \otimes \mathcal{R}$ to $\mathcal{R}[\lambda]=\mathbb{C}[\lambda] \otimes \mathcal{R}$ by $\left[a_{\lambda} b\right]^{\prime}=\left[\alpha(a)_{\lambda}\right.$ $\beta(b)]$. Then $\left(\mathcal{R},\left[\cdot_{\lambda} \cdot\right]^{\prime}, \alpha, \beta\right)$ is a BiHom-Lie conformal superalgebra.

Proof. We will just verify axioms (2.4) and (2.5). Let $a, b, c \in \mathcal{R}$. Then

$$
\left[\beta(a)_{\lambda} \alpha(b)\right]^{\prime}=\left[\alpha \beta(a)_{\lambda} \alpha \beta(b)\right]=-\left[\alpha \beta(b)_{-\lambda-\partial} \alpha \beta(a)\right]=-\left[\beta(b)_{-\lambda-\partial} \alpha(a)\right]^{\prime} .
$$

On the other hand, we have

$$
\begin{aligned}
{\left[\alpha \beta(a)_{\lambda}\left[b_{\mu} c\right]^{\prime}\right]^{\prime} } & =\left[\alpha^{2} \beta(a)_{\lambda}\left[\alpha \beta(b)_{\mu} \beta^{2}(c)\right]\right] \\
& =\left[\left[\alpha^{2} \beta(a)_{\lambda} \alpha \beta(b)\right]_{\lambda+\mu} \beta^{2}(c)\right]+(-1)^{|a||b|}\left[\alpha \beta(b)_{\mu}\left[\alpha^{2} \beta(a)_{\lambda} \beta^{2}(c)\right]\right] \\
& =\left[\left[\beta(a)_{\lambda} b\right]_{\lambda+\mu}^{\prime} \beta(c)\right]^{\prime}+(-1)^{|a||b|}\left[\beta(b)_{\mu}\left[\alpha(a)_{\lambda} c\right]^{\prime}\right]^{\prime}
\end{aligned}
$$

The following result can be proved similarly to the previous one. 
Proposition 2.2. Let $(\mathcal{R},[\cdot \lambda \cdot], \alpha, \beta)$ be a BiHom-Lie conformal superalgebra. Let $\alpha^{\prime}, \beta^{\prime}: \mathcal{R} \rightarrow \mathcal{R}$ be two even homomorphisms of conformal algebras such that any two of the maps $\alpha, \beta, \alpha^{\prime}, \beta^{\prime}$ commute. Then $\left(\mathcal{R},\left[\cdot_{\lambda} \cdot\right]^{\prime}=\left[\cdot_{\lambda} \cdot\right] \circ\left(\alpha^{\prime} \otimes \beta^{\prime}\right), \alpha \circ \alpha^{\prime}, \beta \circ \beta^{\prime}\right)$ is a BiHom-Lie conformal superalgebra.

Corollary 2.1. Let $\left(\mathcal{R},\left[{ }_{\lambda} \cdot\right], \alpha, \beta\right)$ be a BiHom-Lie conformal superalgebra. Then $\left(\mathcal{R},\left[{ }_{\lambda} \cdot\right]^{\prime}=\left[{ }_{\lambda} \cdot\right] \circ\left(\alpha^{k} \otimes \beta^{k}\right), \alpha^{k+1}, \beta^{k+1}\right)$ is a BiHom-Lie conformal superalgebra.

Proposition 2.3. Let $(\mathcal{R}, \alpha, \beta)$ and $\left(\mathcal{R}^{\prime}, \alpha^{\prime}, \beta^{\prime}\right)$ be two BiHom-Lie conformal superalgebras, Then $\left(\mathcal{R} \oplus \mathcal{R}^{\prime}, \alpha+\alpha^{\prime}, \beta+\beta^{\prime}\right)$ is a BiHom-Lie conformal superalgebra with

$$
\begin{aligned}
& {\left[\left(u_{1}+v_{1}\right)_{\lambda}\left(u_{2}+v_{2}\right)\right]=\left[u_{1 \lambda} v_{1}\right]+\left[u_{2 \lambda} v_{2}\right], \forall u_{1}, u_{2} \in \mathcal{R}, \forall v_{1}, v_{2} \in \mathcal{R}^{\prime},} \\
& \left(\alpha+\alpha^{\prime}\right)(u+v)=\alpha(u)+\alpha^{\prime}(v),\left(\beta+\beta^{\prime}\right)(u+v)=\beta(u)+\beta^{\prime}(v), \forall u \in \mathcal{R}, \forall v \in \mathcal{R}^{\prime} .
\end{aligned}
$$

Proof. Straightforward.

Definition 2.2. Let $(M, \alpha, \beta)$ and $(N, \phi, \psi)$ be $\mathbb{Z}_{2}$-graded $\mathbb{C}[\partial]$-modules. A BiHom-conformal linear map of degree $\theta$ from $M$ to $N$ is a sequence $f=f_{(n) n \in \mathbb{Z}_{+}}$of $f_{(n)} \in \operatorname{Hom}_{\mathbb{C}}(M, N)$ satisfying that

$$
\begin{aligned}
& \partial_{N} f_{(n)}-f_{(n)} \partial_{M}=-n f_{(n-1)}, f_{\lambda}\left(M_{\mu}\right) \subseteq N_{\mu+\theta}, n \in \mathbb{Z}_{+}, \mu, \theta \in \mathbb{Z}_{2}, \\
& \partial_{M} \alpha=\alpha \partial_{M}, \partial_{M} \beta=\beta \partial_{M}, \partial_{N} \phi=\phi \partial_{N}, \partial_{N} \psi=\psi \partial_{N} \\
& f_{(n)} \alpha=\phi f_{(n)}, f_{(n)} \beta=\psi f_{(n)} .
\end{aligned}
$$


Set $f_{\lambda}=\sum_{n=0}^{\infty} \frac{\lambda^{n}}{n !} f_{(n)}$. Then $f$ is a BiHom-conformal linear map of degree $\theta$ if and only if

$$
\begin{aligned}
& f_{\lambda} \partial_{M}=\left(\partial_{N}+\lambda\right) f_{\lambda}, f_{\lambda}\left(M_{\mu}\right) \subseteq N_{\mu+\theta}[\lambda], \\
& \partial_{M} \alpha=\alpha \partial_{M}, \partial_{M} \beta=\beta \partial_{M}, \partial_{N} \phi=\phi \partial_{N}, \partial_{N} \psi=\psi \partial_{N}, \\
& f_{\lambda} \alpha=\phi f_{\lambda}, f_{\lambda} \beta=\psi f_{\lambda} .
\end{aligned}
$$

Let $\operatorname{Chom}(M, N)_{\theta}$ denote the set of BiHom-conformal linear maps of degree $\theta$ from $M$ to $N$. Then $\operatorname{Chom}(M, N)=\operatorname{Chom}(M, N)_{0} \oplus \operatorname{Chom}$ $(M, N)_{1}$ is a $\mathbb{Z}_{2}$-graded $\mathbb{C}[\partial]$-module via:

$$
\partial f_{(n)}=-n f_{(n-1)} \text {, equivalently, } \partial f_{\lambda}=-\lambda f_{\lambda} .
$$

The composition $f_{\lambda} g: M \rightarrow N \otimes \mathbb{C}[\lambda]$ of BiHom-conformal linear maps $f: M \rightarrow N$ and $g: L \rightarrow M$ is given by

$$
\left(f_{\lambda} g\right)_{\lambda+\mu}=f_{\lambda} g_{\mu}, \forall f, g \in \operatorname{Chom}(M, N) .
$$

If $M$ is a finitely generated $\mathbb{Z}_{2}$-graded $\mathbb{C}[\partial]$-module, then $\operatorname{Cend}(M):=\operatorname{Chom}(M, M)$ is an associative conformal superalgebra with respect to the above composition. Thus, $\operatorname{Cend}(M)$ becomes a Lie conformal superalgebra, denoted as $g c(M)$, with respect to the following $\lambda$-bracket:

$$
\left[f_{\lambda} g\right]_{\mu}=f_{\lambda} g_{\mu-\lambda}-(-1)^{|f||g|} g_{\mu-\lambda} f_{\lambda}
$$

Hereafter all $\mathbb{Z}_{2}$-graded $\mathbb{C}[\partial]$-module are supposed to be finitely generated. 
Proposition 2.4. Let $(\mathcal{R}, \alpha, \beta)$ be a regular BiHom-associative conformal superalgebra with two even morphisms $\alpha$ and $\beta$. Then the $\lambda$-bracket

$$
\left[a_{\lambda} b\right]=a_{\lambda} b-(-1)^{|a||b|} \alpha^{-1} \beta(b)_{-\lambda-\lambda} \alpha \beta^{-1}(a), \forall a, b \in \mathcal{R} .
$$

defines a BiHom-Lie conformal superalgebra structure on $\mathcal{R}$.

Proof. For any $a, b \in \mathcal{R}$, we have

$$
\begin{aligned}
{\left[\beta(a)_{\lambda} \alpha(b)\right] } & =\beta(a)_{\lambda} \alpha(b)-(-1)^{|a||b|} \beta(b)_{-\lambda-\lambda} \alpha(a) \\
& =-(-1)^{|a||b|}\left(\beta(b)_{-\lambda-\lambda} \alpha(a)-(-1)^{|a||b|} \beta(a)_{\lambda} \alpha(b)\right) \\
& =-(-1)^{|a||b|}\left[\beta(b)_{\lambda} \alpha(a)\right] .
\end{aligned}
$$

On the other hand, given $a, b, c \in \mathcal{R}$. Then

$$
\begin{aligned}
{\left[\alpha \beta(a)_{\lambda}\left[b_{\mu} c\right]\right]=} & {\left[\alpha \beta(a)_{\lambda}\left(b_{\mu} c-(-1)^{|b||c|} \alpha^{-1} \beta(c)_{-\mu-\partial} \alpha \beta^{-1}(b)\right)\right] } \\
= & \alpha \beta(a)_{\lambda}\left(b_{\mu} c\right)-(-1)^{|\alpha|(|b|+|c|)} a^{-1} \beta\left(b_{\mu} c\right)_{-\lambda-\partial} \alpha^{2}(a) \\
& -(-1)^{|b||c|} \alpha \beta(a)_{\lambda}\left(\alpha^{-1} \beta(c)_{-\mu-\partial} \alpha \beta^{-1}(b)\right) \\
& +(-1)^{|a||c|+|\alpha||b|+|b||c|} \alpha^{-1} \beta\left(\alpha^{-2} \beta^{2}(c)_{-\mu-\partial} b\right)_{-\lambda-\partial} \alpha^{2}(a) .
\end{aligned}
$$

Similarly, we have

$$
\begin{aligned}
& {\left[\left[\beta(a)_{\lambda} b\right]_{\lambda+\mu} \beta(c)\right] } \\
&= {\left[\left(\beta(a)_{\lambda} b-(-1)^{|\alpha||b|} \alpha^{-1} \beta(b)_{-\lambda-\lambda} \alpha(a)\right)_{\lambda+\mu} \beta(c)\right] } \\
&=\left(\beta(a)_{\lambda} b\right)_{\lambda+\mu} \beta(c)-(-1)^{|c|(|a|+|c|)} \alpha^{-1} \beta^{2}(c)_{-\lambda-\mu-\partial}\left(\alpha(a)_{\lambda} \alpha \beta^{-1}(b)\right) \\
&-(-1)^{|\alpha||b|}\left(\alpha^{-1} \beta(b)_{-\lambda-\lambda} \alpha(a)\right)_{\lambda+\mu} \beta(c) \\
&+(-1)^{|a||c|+|a||b|+|b||c|} \alpha^{-1} \beta^{2}(c)_{-\lambda-\mu-\partial}\left(b_{-\lambda-\partial} \alpha^{2} \beta^{-1}(a)\right),
\end{aligned}
$$


and

$$
\begin{aligned}
&(-1)^{|a||b|}\left[\beta(b)_{\mu},\left[\alpha(a)_{\lambda} c\right]\right] \\
&=(-1)^{|\alpha||b|} \beta(b)_{\mu}\left(\alpha(a)_{\lambda} c\right)-(-1)^{|a|(|c|+|b|)} \beta(b)_{\mu}\left(\alpha^{-1} \beta(c)_{-\lambda-\partial} \alpha^{2} \beta^{-1}(a)\right) \\
&-(-1)^{|\alpha||c|}\left(\beta(a)_{\lambda} \alpha^{-1} \beta(c)\right)_{-\mu-\partial} \alpha(b) \\
&+(-1)^{|a| b \mid}\left(\alpha^{-2} \beta^{2}(c)_{-\lambda-\lambda} \alpha(a)\right)_{-\mu-\partial} \alpha(b) .
\end{aligned}
$$

Using the associativity, it is not hard to check that

$$
\left[\alpha \beta(a)_{\lambda}\left[b_{\mu} c\right]\right]=\left[\left[\beta(a)_{\lambda} b\right]_{\lambda+\mu} \beta(c)\right]+(-1)^{|a||b|}\left[\beta(b)_{\mu}\left[\alpha(a)_{\lambda} c\right]\right]
$$

as desired. This finishes the proof.

\section{Cohomology of BiHom-Lie Conformal Superalgebra}

In this section, we develop representation and cohomology theory of BiHom-Lie conformal superalgebras.

Definition 3.1. A $\mathbb{C}[\partial]$-module $(M, \phi, \psi)$ is a BiHom-conformal module of a BiHom-Lie conformal superalgebra $\mathcal{R}$ if there is a $\mathbb{C}$-linear map $\rho: \mathcal{R} \rightarrow \operatorname{Cend}(M)$ satisfying the following conditions (for all $a, b \in \mathcal{R}$ ):

$$
\begin{gathered}
\rho(\partial(a))_{\lambda}=-\lambda \rho(a)_{\lambda}, \rho(a)_{\lambda} \partial=(\lambda+\partial) \rho(a)_{\lambda}, \\
\phi \psi=\psi \phi, \psi \partial=\partial \psi, \phi \partial=\partial \phi \\
\phi \rho(a)_{\lambda}=\rho(\alpha(a))_{\lambda} \phi, \psi \rho(a)_{\lambda}=\rho(\beta(a))_{\lambda} \psi, \\
\rho\left(\left[\beta(a)_{\lambda} b\right]\right)_{\lambda+\mu} \psi=\rho(\alpha \beta(a))_{\lambda} \rho(b)_{\mu}-(-1)^{|a||b|} \rho(\beta(b))_{\mu} \rho(\alpha(a))_{\lambda} .
\end{gathered}
$$

The map $\rho$ is called the corresponding representation. 
Example 3.1. Let $(\mathcal{R}, \alpha, \beta)$ be a BiHom-Lie conformal superalgebra. Then $(\mathcal{R}, \alpha, \beta)$ is an $\mathcal{R}$-module under the adjoint diagonal action, $\rho(a)_{\lambda} b=\left[a_{\lambda} b\right], \forall a, b \in \mathcal{R}$.

Example 3.2. Let $(g,[\because],, \alpha, \beta)$ be a finite BiHom-Lie superalgebra, $(M, \phi, \psi)$ be a BiHom-conformal module of $g$ with corresponding representation map $\varrho: g \rightarrow g l(M)$. Then the $\mathbb{Z}_{2}$-graded $\mathbb{C}[\partial]$-module $(\mathbb{C}[\partial] \otimes M, \tilde{\phi}, \widetilde{\psi})$ is a BiHom-conformal module of Cur $g$, where

$$
\widetilde{\phi}(f(\partial) \otimes m)=f(\partial) \otimes \phi(m), \widetilde{\psi}(f(\partial) \otimes m)=f(\partial) \otimes \psi(m),
$$

with a module structure $\rho: \operatorname{Cur} g \rightarrow \operatorname{Cend}(\mathbb{C}[\partial] \otimes M)$ given by $\rho(f(\partial) \otimes a)_{\lambda}(g(\partial) \otimes m)=f(-\partial) g(\partial+\lambda) \otimes \varrho(a)(m), \forall f, g \in \mathbb{C}[\partial], a \in g, m \in M$.

Proposition 3.1. Let $(\mathcal{R}, \alpha, \beta)$ be a regular BiHom-Lie conformal superalgebra, $(M, \phi, \psi)$ a BiHom-conformal module of $\mathcal{R}$ and $\rho: \mathcal{R} \rightarrow$ $\mathbb{C}[\lambda] \otimes \operatorname{Cend}(M): a \mapsto \rho(a)_{\lambda}$ the corresponding representation. Define a $\lambda$-bracket $\left[-\lambda^{-}\right]_{M}$ on $\mathcal{R} \oplus M=(\mathcal{R} \oplus M)_{0} \oplus(\mathcal{R} \oplus M)_{1}$ by

$$
\left[(r+m)_{\lambda}\left(r^{\prime}+m^{\prime}\right)\right]_{M}=\left[r_{\lambda} r^{\prime}\right]+\rho(r)_{\lambda} m^{\prime}-(-1)^{\left|r^{\prime}\right||m|} \rho\left(\alpha^{-1} \beta\left(r^{\prime}\right)\right)_{-\partial-\lambda} \phi \psi^{-1}(m),
$$

$\forall r+m, r^{\prime}+m^{\prime} \in \mathcal{R} \oplus M$, where $(\mathcal{R} \oplus M)_{\theta}=\mathcal{R}_{\theta} \oplus M_{\theta}, \theta \in \mathbb{Z}_{2}$. Then $(\mathcal{R} \oplus M, \alpha+\phi, \beta+\psi)$ is a BiHom-Lie conformal superalgebra, called the semidirect product of $\mathcal{R}$ and $M$, and denote by $\mathcal{R} \ltimes_{\rho} M$.

Proof. $\forall r+m, r^{\prime}+m^{\prime}, r^{\prime \prime}+m^{\prime \prime} \in \mathcal{R} \oplus M$, note that $\mathcal{R} \oplus M$ is equipped with a $\mathbb{C}[\partial]$-module structure via

$$
\partial(r+m)=\partial(r)+\partial(m)
$$


In addition, it is immediate that $\partial(\alpha+\phi)=(\alpha+\phi) \partial$ and $\partial(\beta+\psi)=(\beta+\psi) \partial$.

A direct computation gives

$$
\begin{aligned}
& {\left[\partial(r+m)_{\lambda}\left(r^{\prime}+m^{\prime}\right)\right]_{M}=\left[(\partial r+\partial m)_{\lambda}\left(r^{\prime}+m^{\prime}\right)\right]_{M}} \\
& \quad=\left[(\partial r)_{\lambda} r^{\prime}\right]+\rho(\partial r)_{\lambda}\left(m^{\prime}\right)-(-1)^{\left|r^{\prime}\right||m|} \rho\left(\alpha^{-1} \beta\left(r^{\prime}\right)\right)_{-\partial-\lambda}\left(\partial \phi \psi^{-1}(m)\right) \\
& \quad=-\lambda\left[r_{\lambda} r^{\prime}\right]-\lambda \rho(r)_{\lambda}\left(m^{\prime}\right)-(-1)^{\left|r^{\prime}\right||m|}(\partial-\lambda-\partial) \rho\left(\alpha^{-1} \beta\left(r^{\prime}\right)\right)_{-\partial-\lambda}\left(\phi \psi^{-1}(m)\right) \\
& \quad=-\lambda\left(\left[r_{\lambda} r^{\prime}\right]+\rho(r)_{\lambda}\left(m^{\prime}\right)-(-1)^{\left|r^{\prime}\right||m|} \rho\left(\alpha^{-1} \beta\left(r^{\prime}\right)\right)_{-\partial-\lambda}\left(\phi \psi^{-1}(m)\right)\right) \\
& \quad=-\lambda\left[(r+m)_{\lambda}\left(r^{\prime}+m^{\prime}\right)\right]_{M},
\end{aligned}
$$

and

$$
\begin{aligned}
& {\left[(r+m)_{\lambda} \partial\left(r^{\prime}+m^{\prime}\right)\right]_{M}=\left[(r+m)_{\lambda}\left(\partial r^{\prime}+\partial m^{\prime}\right)\right]_{M}} \\
& \quad=\left[r_{\lambda} \partial r^{\prime}\right]+\rho(r)_{\lambda}\left(\partial m^{\prime}\right)-(-1)^{\left|r^{\prime}\right||m|} \rho\left(\partial \alpha^{-1} \beta\left(r^{\prime}\right)\right)_{-\lambda-\partial} \phi \psi^{-1}(m) \\
& \quad=(\lambda+\partial)\left(\left[r_{\lambda} r^{\prime}\right]+\rho(r)_{\lambda}\left(m^{\prime}\right)-(-1)^{\left|r^{\prime}\right||m|} \rho\left(\alpha^{-1} \beta\left(r^{\prime}\right)\right)_{-\lambda-\partial} \phi \psi^{-1}(m)\right) \\
& \quad=(\lambda+\partial)\left[(r+m)_{\lambda}\left(r^{\prime}+m^{\prime}\right)\right]_{M} .
\end{aligned}
$$

Thus (2.3) holds. (2.4) follows from

$$
\begin{aligned}
{[(\beta(r)+} & \left.\psi(m))_{\lambda}\left(\alpha\left(r^{\prime}\right)+\phi\left(m^{\prime}\right)\right)\right]_{M} \\
= & {\left[\beta(r)_{\lambda} \alpha\left(r^{\prime}\right)\right]+\rho(\beta(r))_{\lambda}\left(\phi\left(m^{\prime}\right)\right)-(-1)^{\left|r^{\prime}\right||m|} \rho\left(\beta\left(r^{\prime}\right)\right)_{-\partial-\lambda}(\phi(m)) } \\
= & -(-1)^{\left|r^{\prime}\right||m|}\left(\left[\beta\left(r^{\prime}\right)_{-\partial-\lambda} \alpha(r)\right]+\rho\left(\beta\left(r^{\prime}\right)\right)_{-\partial-\lambda}(\phi(m))\right. \\
& -(-1)^{|r||m|} \rho(\beta(r))_{-\partial-(-\partial-\lambda)}\left(\phi\left(m^{\prime}\right)\right) \\
= & -(-1)^{\left|r^{\prime}\right||m|}\left[\left(\beta\left(r^{\prime}\right)+\psi\left(m^{\prime}\right)\right)_{-\partial-\lambda}(\alpha(r)+\phi(m))\right]_{M} .
\end{aligned}
$$


To check the BiHom-Jacobi conformal identity (2.5), we compute

$$
\begin{aligned}
{\left[(\alpha \beta(r)+\phi \psi(m))_{\lambda}\left[\left(r^{\prime}+m^{\prime}\right)_{\mu}\left(r^{\prime \prime}+m^{\prime \prime}\right)\right]_{M}\right]_{M} } & \\
= & {\left[( \alpha \beta ( r ) + \phi \psi ( m ) ) _ { \lambda } \left(\left[r_{\mu}^{\prime} r^{\prime \prime}\right]+\rho\left(r^{\prime}\right)_{\mu}\left(m^{\prime \prime}\right)\right.\right.} \\
& \left.-(-1)^{\left|r^{\prime \prime}\right|\left|m^{\prime}\right|} \rho\left(\alpha^{-1} \beta\left(r^{\prime \prime}\right)\right)_{-\partial-\mu}\left(\phi \psi^{-1}\left(m^{\prime}\right)\right)\right]_{M} \\
= & {\left[\alpha \beta(r)_{\lambda}\left[r_{\mu}^{\prime} r^{\prime \prime}\right]\right]+\rho(\alpha \beta(r))_{\lambda} \rho\left(r^{\prime}\right)_{\mu}\left(m^{\prime \prime}\right) } \\
& -(-1)^{\left|r^{\prime \prime}\right|\left|m^{\prime}\right|} \rho(\alpha \beta(r))_{\lambda} \rho\left(\alpha^{-1} \beta\left(r^{\prime \prime}\right)\right)_{-\partial-\mu} \phi \psi^{-1}\left(m^{\prime}\right) \\
& -(-1)^{\left(\left|r^{\prime \prime}\right|+\left|r^{\prime}\right|\right)|m|} \rho\left(\left[\alpha^{-1} \beta\left(r^{\prime}\right)_{\mu} \alpha^{-1} \beta\left(r^{\prime \prime}\right)\right]\right)_{-\partial-\lambda} \phi^{2}(m), \\
(-1)^{|r|\left|r^{\prime}\right|}\left[\left(\beta\left(r^{\prime}\right)+\psi\left(m^{\prime}\right)\right)_{\mu}\left[(\alpha(r)+\phi(m))_{\lambda}\left(r^{\prime \prime}+m^{\prime \prime}\right)\right]_{M}\right]_{M} & \\
= & (-1)^{r|| r^{\prime} \mid}\left[\beta\left(r^{\prime}\right)_{\mu}\left[\alpha(r)_{\lambda} r^{\prime \prime}\right]\right]+(-1)^{|r|\left|r^{\prime}\right|} \rho\left(\beta\left(r^{\prime}\right)\right)_{\mu}\left(\rho(\alpha(r))_{\lambda}\left(m^{\prime \prime}\right)\right) \\
& -(-1)^{|r|\left|r^{\prime}\right|+\left|r^{\prime \prime}\right||m|} \rho\left(\beta\left(r^{\prime}\right)\right)_{\mu}\left(\rho\left(\alpha^{-1} \beta\left(r^{\prime \prime}\right)\right)_{-\partial-\lambda}\left(\phi^{2} \psi^{-1}(m)\right)\right) \\
& -(-1)^{\left|r^{\prime \prime}\right|\left|m^{\prime}\right|} \rho\left(\left[\beta(r)_{\lambda} \alpha^{-1} \beta\left(r^{\prime \prime}\right)\right]\right)_{-\partial-\mu} \phi\left(m^{\prime}\right),
\end{aligned}
$$

and

$$
\begin{aligned}
{[[\beta(r)+} & \left.\left.\psi(m))_{\lambda}\left(r^{\prime}+m^{\prime}\right)\right]_{M_{(\lambda+\mu)}}\left(\beta\left(r^{\prime \prime}\right)+\psi\left(m^{\prime \prime}\right)\right)\right]_{M} \\
= & {\left[\left(\left[\beta(r)_{\lambda} r^{\prime}\right]+\rho(\beta(r))_{\lambda}\left(m^{\prime}\right)-(-1)^{\left|r^{\prime}\right||m|} \rho\left(\alpha^{-1} \beta\left(r^{\prime}\right)\right)_{-\partial-\lambda}\right.\right.} \\
& \left.\left.\times \phi \psi^{-1}(m)\right)_{\lambda+\mu}\left(\beta\left(r^{\prime \prime}\right)+\psi\left(m^{\prime \prime}\right)\right)\right]_{M} \\
= & {\left[\left[\beta(r)_{\lambda} \beta\left(r^{\prime}\right)\right]_{\lambda+\mu} r^{\prime \prime}\right]+\rho\left(\left[\beta(r)_{\lambda} r^{\prime}\right]\right)_{\lambda+\mu}\left(\psi\left(m^{\prime \prime}\right)\right) } \\
& -(-1)^{\left|r^{\prime \prime}\right|\left(|r|+\left|m^{\prime}\right|\right)} \rho\left(\alpha^{-1} \beta^{2}\left(r^{\prime \prime}\right)\right)_{-\partial-\lambda-\mu} \phi \psi^{-1}\left(\rho(\beta(r))_{\lambda} m^{\prime}\right) \\
& +(-1)^{\left|r^{\prime \prime}\right|\left(\left|r^{\prime}\right|+|m|\right)+\left|r^{\prime}\right||m|} \rho\left(\alpha^{-1} \beta^{2}\left(r^{\prime \prime}\right)\right)_{-\partial-\lambda-\mu} \phi \psi^{-1}\left(\rho\left(\alpha^{-1} \beta\left(r^{\prime}\right)\right)_{-\partial-\lambda} \phi(m)\right) .
\end{aligned}
$$


Now, since $(M, \rho, \phi, \psi)$ is a representation of $\mathcal{R}$, then we can easily conclude that $\mathcal{R} \oplus M$ is a BiHom-Lie conformal superalgebra.

In the following we aim to develop the cohomology theory of regular BiHom-Lie conformal superalgebras. To do this, we need the following concept.

Definition 3.2. An $n$-cochain $\left(n \in \mathbb{Z}_{+}\right)$of a BiHom-Lie conformal superalgebra $\mathcal{R}$ with coefficients in a module $M$ is a $\mathbb{C}$-linear map of degree $\theta$

$$
\begin{gathered}
\gamma: \mathcal{R}^{n} \rightarrow M\left[\lambda_{1}, \cdots, \lambda_{n}\right], \\
\left(a_{1}, \cdots, a_{n}\right) \mapsto \gamma_{\lambda_{1}, \cdots, \lambda_{n}}\left(a_{1}, \cdots, a_{n}\right),
\end{gathered}
$$

where $M\left[\lambda_{1}, \cdots, \lambda_{n}\right]$ denotes the space of polynomials with coefficients in $M$, satisfying the following conditions:

Conformal antilinearity:

$$
\gamma_{\lambda_{1}, \cdots, \lambda_{n}}\left(a_{1}, \cdots, \partial a_{i}, \cdots, a_{n}\right)=-\lambda_{i} \gamma_{\lambda_{1}, \cdots, \lambda_{n}}\left(a_{1}, \cdots, a_{i}, \cdots, a_{n}\right) .
$$

Skew-symmetry:

$$
\begin{aligned}
& \gamma_{\lambda_{1}, \cdots, \lambda_{i}, \lambda_{i+1}, \cdots, \lambda_{n}}\left(a_{1}, \cdots, \beta\left(a_{i}\right), \alpha\left(a_{i+1}\right), \cdots, a_{n}\right) \\
& =-(-1)^{\left|a_{i}\right|\left|a_{i+1}\right|} \gamma_{\lambda_{1}, \cdots, \lambda_{i+1}, \lambda_{i}, \cdots, \lambda_{n}}\left(a_{1}, \cdots, \beta\left(a_{i+1}\right), \alpha\left(a_{i}\right), \cdots, a_{n}\right) .
\end{aligned}
$$

Commutativity: $\gamma \circ \alpha=\phi \circ \gamma, \gamma \circ \beta=\psi \circ \gamma$.

A 0-cochain is just an element in $M$. Define a differential $d$ of a cochain $\gamma$ by

$$
\begin{aligned}
& (d \gamma)_{\lambda_{1}, \cdots, \lambda_{n+1}}\left(a_{1}, \cdots, a_{n+1}\right) \\
& =\sum_{i=1}^{n+1}(-1)^{i+1}(-1)^{\left(|\gamma|+\left|a_{1}\right|+\cdots+\left|a_{i-1}\right|\right)\left|a_{i}\right|} \rho\left(\alpha \beta^{n-1}\left(a_{i}\right)\right)_{\lambda_{i}} \\
& \quad \times \gamma_{\lambda_{1}, \cdots, \hat{\lambda}_{i}, \cdots, \lambda_{n+1}}\left(a_{1}, \cdots, \hat{a}_{i}, \cdots, a_{n+1}\right)
\end{aligned}
$$




$$
\begin{aligned}
& +\sum_{1 \leq i<j}^{n+1}(-1)^{i+j}(-1)^{\left(\left|a_{1}\right|+\cdots+\left|a_{i-1}\right|\right)\left|a_{i}\right|+\left(\left|a_{1}\right|+\cdots+\left|a_{j-1}\right|\right)\left|a_{j}\right|+\left|a_{i}\right|\left|a_{j}\right|} \\
& \times \gamma_{\lambda_{i}+\lambda_{j}, \lambda_{1}, \cdots, \hat{\lambda}_{i}, \cdots, \hat{\lambda}_{j}, \cdots, \lambda_{n+1}}\left(\left[\alpha^{-1} \beta\left(a_{i}\right)_{\lambda_{i}} a_{j}\right],\right. \\
& \left.\beta\left(a_{1}\right), \cdots, \hat{a}_{i}, \cdots, \hat{a}_{j}, \cdots, \beta\left(+a_{n+1}\right)\right),
\end{aligned}
$$

where $\rho$ is the corresponding representation of $M$, and $\gamma$ is extended linearly over the polynomials in $\lambda_{i}$. In particular, if $\gamma$ is a 0-cochain, then $(d \gamma)_{\lambda} a=a_{\lambda} \gamma$.

Proposition 3.2. $d \gamma$ is a cochain and $d^{2}=0$.

Proof. Let $\gamma$ be an $n$-cochain. It is not hard to see that $d \gamma$ satisfies conformal antilinearity, skew-symmetry and commutativity. That is, $d \gamma$ is an $(n+1)$-cochain. It remains to show that $d^{2}=0$. In fact, we have

$$
\begin{aligned}
& \left(d^{2} \gamma\right)_{\lambda_{1}, \ldots, \lambda_{n+2}}\left(a_{1}, \ldots, a_{n+2}\right) \\
& =\sum_{i=1}^{n+1}(-1)^{i+1}(-1)^{i+1+|\gamma|\left|a_{i}\right|+A_{i}} \rho\left(\alpha \beta^{n}\left(a_{i}\right)\right)_{\lambda_{i}}(d \gamma)_{\lambda_{1}, \ldots, \lambda_{i}, \ldots, \lambda_{n+2}} \\
& \times\left(a_{1}, \ldots, \hat{a}_{i}, \ldots, a_{n+2}\right) \\
& +\sum_{1 \leq i<j \leq n+1}(-1)^{i+j+A_{i}+A_{j}+\left|a_{i}\right|\left|a_{j}\right|}(d \gamma)_{\lambda_{i}+\lambda_{j}, \lambda_{1}, \ldots, \hat{\lambda}_{i}, \ldots \hat{\lambda}_{j}, \ldots, \lambda_{n+2}} \\
& \times\left(\left[\alpha^{-1} \beta\left(a_{i}\right)_{\lambda_{i}} a_{j}\right], \beta\left(a_{1}\right), \ldots, \hat{a}_{i}, \ldots, \hat{a}_{j}, \ldots, \beta\left(a_{n+2}\right)\right) \\
& =\sum_{i=1}^{n+2} \sum_{j=1}^{i-1}(-1)^{i+j+|\gamma|\left(\left|a_{i}\right|+\left|a_{j}\right|\right)+A_{i}+A_{j}} \rho\left(\alpha \beta^{n}\left(a_{i}\right)\right)_{\lambda_{i}}\left(\alpha \beta^{n-1}\left(a_{j}\right)_{\lambda_{j}}\right. \\
& \gamma_{\lambda_{1}, \ldots, \hat{\lambda}_{j, i}, \ldots, \lambda_{n+2}}\left(a_{1}, \ldots, \hat{a}_{j, i}, \ldots, a_{n+2}\right)
\end{aligned}
$$




$$
\begin{aligned}
& \sum_{i=1}^{n+2} \sum_{j=i+1}^{n+2}(-1)^{i+j+1+|\gamma|\left(\left|a_{i}\right|+\left|a_{j}\right|\right)+A_{i}+\left(A_{j}-\left|a_{i}\right|\right)} \rho\left(\alpha \beta^{n}\left(a_{i}\right)\right)_{\lambda_{i}} \\
& \left(\alpha \beta^{n-1}\left(a_{j}\right)_{\lambda_{j}} \gamma_{\lambda_{1}, \ldots, \hat{\lambda}_{i, j}, \ldots, \lambda_{n+2}}\left(a_{1}, \ldots, \hat{a}_{i, j}, \ldots, a_{n+2}\right)\right. \\
& +\sum_{i=1}^{n+2} \sum_{1 \leq j<k<i}^{n+2}(-1)^{i+j+k+1+|\gamma|\left|a_{i}\right|+A_{i}+A_{j}+A_{k}+\left|a_{j}\right|\left|a_{k}\right|} \rho\left(\alpha \beta^{n}\left(a_{i}\right)\right)_{\lambda_{i}} \\
& \gamma_{\lambda_{j}+\lambda_{k}, \lambda_{1}, \ldots, \hat{\lambda}_{j, k, i}, \ldots, \lambda_{n+2}}\left(\left[\alpha^{-1} \beta\left(a_{j}\right)_{\lambda_{j}} a_{k}\right], \beta\left(a_{1}\right), \ldots, \hat{a}_{j, k, i}, \ldots, \beta\left(a_{n+2}\right)\right) \\
& +\sum_{i=1}^{n+2} \sum_{1 \leq j<i<k}^{n+2}(-1)^{i+j+k+|\gamma|\left|a_{i}\right|+A_{i}+A_{j}+\left(A_{k}-\left|a_{i}\right|\right)+\left|a_{j}\right|\left|a_{k}\right|} \rho\left(\alpha \beta^{n}\left(a_{i}\right)\right)_{\lambda_{i}} \\
& \gamma_{\lambda_{j}+\lambda_{k}, \lambda_{1}, \ldots, \hat{\lambda}_{j, i, k}, \ldots, \lambda_{n+2}}\left(\left[\alpha^{-1} \beta\left(a_{j}\right)_{\lambda_{j}} a_{k}\right], \beta\left(a_{1}\right), \ldots, \hat{a}_{j, i, k}, \ldots, \beta\left(a_{n+2}\right)\right)
\end{aligned}
$$

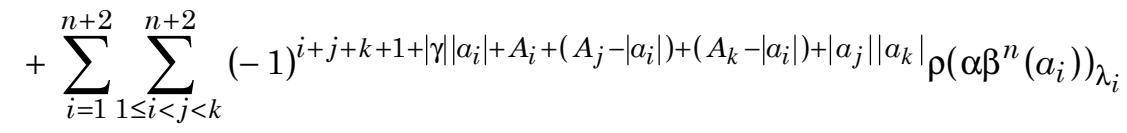

$$
\begin{aligned}
& \gamma_{\lambda_{j}+\lambda_{k}, \lambda_{1}, \ldots, \hat{\lambda}_{i, j, k}, \ldots, \lambda_{n+2}}\left(\left[\alpha^{-1} \beta\left(a_{j}\right)_{\lambda_{j}} a_{k}\right], \beta\left(a_{1}\right), \ldots, \hat{a}_{i, j, k}, \ldots, \beta\left(a_{n+2}\right)\right) \\
& +\sum_{1 \leq i<j}^{n+2} \sum_{k=1}^{i-1}(-1)^{i+j+k+A_{i}+A_{j}+A_{k}+\left|a_{i}\right|\left|a_{j}\right|+\left(|\gamma|+\left|a_{i}\right|+\left|a_{j}\right|\right)\left|a_{k}\right|} \rho\left(\alpha \beta^{n}\left(a_{k}\right)\right)_{\lambda_{k}} \\
& \gamma_{\lambda_{i}+\lambda_{j}, \lambda_{1}, \ldots, \hat{\lambda}_{k, i, j}, \ldots, \lambda_{n+2}}\left(\left[\alpha^{-1} \beta\left(a_{i}\right)_{\lambda_{i}} a_{j}\right], \beta\left(a_{1}\right), \ldots, \hat{a}_{k, i, j}, \ldots, \beta\left(a_{n+2}\right)\right)
\end{aligned}
$$




$$
\begin{aligned}
& +\sum_{1 \leq i<j}^{n+2} \sum_{k=i+1}^{j-1}(-1)^{i+j+k+1+A_{i}+A_{j}+A_{k}+\left|a_{i}\right|\left|a_{j}\right|+\left(|\gamma|+\left|a_{j}\right|\right)\left|a_{k}\right|} \rho\left(\alpha \beta^{n}\left(a_{k}\right)\right)_{\lambda_{k}} \\
& \gamma_{\lambda_{i}+\lambda_{j}, \lambda_{1}, \ldots, \hat{\lambda}_{i, k, j}, \ldots, \lambda_{n+2}}\left(\left[\alpha^{-1} \beta\left(a_{i}\right)_{\lambda_{j}} a_{j}\right], \beta\left(a_{1}\right), \ldots, \hat{a}_{i, k, j}, \ldots, \beta\left(a_{n+2}\right)\right)
\end{aligned}
$$

$$
\begin{aligned}
& +\sum_{1 \leq i<j}^{n+2} \sum_{k=j+1}^{n+2}(-1)^{i+j+k+A_{i}+A_{j}+A_{k}+\left|a_{i}\right|\left|a_{j}\right|+|\gamma|\left|a_{k}\right|} \rho\left(\alpha \beta^{n}\left(a_{k}\right)\right)_{\lambda_{k}} \\
& \gamma_{\lambda_{i}+\lambda_{j}, \lambda_{1}, \ldots, \hat{\lambda}_{i, j, k}, \ldots, \lambda_{n+2}}\left(\left[\alpha^{-1} \beta\left(a_{i}\right)_{\lambda_{i}} a_{j}\right], \beta\left(a_{1}\right), \ldots, \hat{a}_{i, j, k}, \ldots, \beta\left(a_{n+2}\right)\right)
\end{aligned}
$$

$$
\begin{aligned}
& +\sum_{1 \leq i<j}^{n+2}(-1)^{i+j+A_{i}+A_{j}+\left|a_{i}\right|\left|a_{j}\right|+|\gamma|\left(\left|a_{i}\right|+\left|a_{j}\right|\right)} \rho\left(\alpha \beta^{n-1}\left(\left[\alpha^{-1} \beta\left(a_{i}\right)_{\lambda_{i}} a_{j}\right]\right)\right)_{\lambda_{i}+\lambda_{j}} \\
& \gamma_{\lambda_{1}, \ldots, \hat{\lambda}_{j}, \ldots, \hat{\lambda}_{i}, \ldots, \lambda_{n+2}}\left(\beta\left(a_{1}\right), \ldots, \hat{a}_{j}, \ldots, \hat{a}_{i}, \ldots, \beta\left(a_{n+2}\right)\right. \\
& +\sum_{\operatorname{distincti}, j, k, l, i<j, k<l}^{n+2}(-1)^{i+j+k+l} \operatorname{sg}\{i, j, k, l\} \\
& (-1)^{A_{i}+A_{j}+\left|a_{i}\right|\left|a_{j}\right|+\left(\left|a_{i}\right|+\left|a_{j}\right|\right)\left(\left|a_{k}\right|+\left|a_{l}\right|\right)} \gamma_{\lambda_{k}+\lambda_{l}, \lambda_{i}+\lambda_{j}, \lambda_{1}, \ldots, \hat{\lambda}_{i, j, k, l}, \ldots, \lambda_{n+2}} \\
& \left(\beta\left[\alpha^{-1} \beta\left(a_{k}\right)_{\lambda_{k}} a_{l}\right], \beta\left[\alpha^{-1} \beta\left(a_{i}\right)_{\lambda_{i}} a_{j}\right], \ldots, \hat{a}_{i, j, k, l}, \ldots, \beta^{2}\left(a_{n+2}\right)\right) \\
& \sum_{i, j, k=1, i<j, k \neq i, j}^{n+2}(-1)^{i+j+k+l} \operatorname{sg}\{i, j, k\}(-1)^{A_{i}+A_{j}+\left|a_{i}\right|\left|a_{j}\right|+\left(A_{k}-\left|a_{i}\right|-\left|a_{j}\right|\right)} \\
& \gamma_{\lambda_{i}+\lambda_{k}+\lambda_{j}, \lambda_{1}, \ldots, \hat{\lambda}_{i, j, k}, \ldots, \lambda_{n+2}}\left(\left[\left[\alpha^{-1} \beta\left(a_{i}\right)_{\lambda_{i}} a_{j}\right]_{\lambda_{i}+\lambda_{j}}, \beta\left(\alpha_{k}\right)\right],\right. \\
& \left.\beta^{2}\left(a_{1}\right), \ldots, \hat{a}_{i, j, k}, \ldots, \beta^{2}\left(a_{n+2}\right)\right),
\end{aligned}
$$

where $\operatorname{sg}\left\{i_{1}, \ldots, i_{p}\right\}$ is the sign of the permutation putting the indices in increasing order and $\hat{a}_{i, j}, a_{i}, a_{j}, \ldots$ are omitted. 
It is obvious that (3.11) and (3.22) summations cancel each other. The same is true for (3.13) and (3.15), (3.13) and (3.14). The BiHom-Jacobi identity implies (3.19) $=0$ and the skew-symmetry implies $(3.18)=0$. Because $(M, \rho, \phi, \psi)$ is an $\mathcal{R}$-module, it follows that

$$
\rho\left(\left[\beta(a)_{\lambda} b\right]\right)_{\lambda+\mu} \psi(v)=\rho(\alpha \beta(a))_{\lambda} \rho(b)_{\mu}(v)-(-1)^{|a||b|} \rho(\beta(b))_{\mu} \rho(\alpha(a)) \lambda(v) .
$$

Since $\gamma \circ \alpha=\alpha_{M} \circ \gamma, \gamma \circ \beta=\beta_{M} \circ \gamma$, we have (3.9), (3.10) and (3.17) summations vanish. Therefore $d^{2} \gamma=0$ and the proof is completed.

Thus the cochains of a BiHom-Lie conformal superalgebra $(\mathcal{R}, \alpha, \beta)$ with coefficients in a module $M$ form a complex, which is denoted by

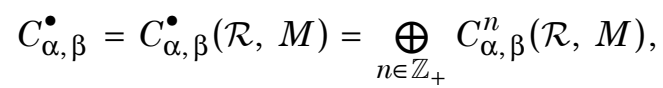

where $C_{\alpha, \beta}^{n}(\mathcal{R}, M)=C_{\alpha, \beta}^{n}(\mathcal{R}, M)_{0} \oplus C_{\alpha, \beta}^{n}(\mathcal{R}, M)_{1}, C_{\alpha, \beta}^{n}(\mathcal{R}, M)_{\theta}$ is the set of $n$-cochain of degree $\theta\left(\theta \in \mathbb{Z}_{2}\right)$. The cohomology group $H_{\alpha, \beta}^{\bullet}(\mathcal{R}, M)$ of a BiHom-Lie conformal superalgebra $\mathcal{R}$ with coefficients in a module $M$ is the cohomology of complex $C_{\alpha, \beta}^{\bullet}$.

Definition 3.3. Let $(\mathcal{R}, \alpha, \beta)$ be a regular BiHom-Lie conformal superalgebra, $(M, \phi, \psi)$ a BiHom-conformal module of $\mathcal{R}$ and $\rho: \mathcal{R} \rightarrow$ $\mathbb{C}[\lambda] \otimes \operatorname{Cend}(M): a \mapsto \rho(a)_{\lambda}$ the corresponding representation. If a $C[\partial]$-module homomorphism $T: M \rightarrow A$ satisfies

$$
\begin{gathered}
T \circ \phi=\alpha \circ T, \quad T \circ \psi=\beta \circ T, \\
{\left[T u_{\lambda} T v\right]=T\left(\rho(T u)_{\lambda} v-(-1)^{|u||v|} \rho\left(T \phi^{-1} \psi(v)\right)_{-\lambda-\partial} \phi \psi^{-1}(u)\right), \forall u, v \in M,}
\end{gathered}
$$

then $T$ is called an $O$-operator associated with $\rho$. 
Proposition 3.3. Let $(\mathcal{R}, \alpha, \beta)$ be a regular BiHom-Lie conformal superalgebra, $(M, \rho, \phi, \psi)$ be a representation and $T: M \rightarrow A$ be an $O$-operator. Then the $\lambda$-product

$$
\left[u_{\lambda} v\right]_{T}=\rho(T u)_{\lambda} v-(-1)^{|u||v|} \rho\left(T \phi^{-1} \psi(v)\right)_{-\lambda-\partial} \phi \psi^{-1}(u), u, v \in M,
$$

defines a BiHom-Lie superalgebra structure on $M$.

Proof. It can be checked by a direct computation, so we omit details.

The following result is obvious.

Corollary 3.1. Let $T$ be an O-operator on a BiHom-Lie superalgebra $(\mathcal{R}, \alpha, \beta)$ with respect to a representation $(M, \rho, \phi, \psi)$. Then $T$ is a homomorphism from the BiHom-Lie superalgebra $\left(M,\left[{ }_{\lambda} \cdot\right]_{T}, \phi, \psi\right)$ to the BiHom-Lie superalgebra $(\mathcal{R}, \alpha, \beta)$.

\section{Generalized Derivations of BiHom-Lie Conformal Superalgebras}

In this section, we investigate derivations and generalized derivations of BiHom-Lie conformal superalgebras and study some of their properties.

Definition 4.1. Let $(\mathcal{R}, \alpha, \beta)$ be a BiHom-Lie conformal superalgebra. Then a BiHom conformal linear map $f_{\lambda}: \mathcal{R} \rightarrow \mathcal{R}$ is called an $\alpha^{k} \beta^{l}$-derivation of $(\mathcal{R}, \alpha, \beta)$ if

$$
\begin{aligned}
& f_{\lambda} \circ \alpha=\alpha \circ f_{\lambda}, f_{\lambda} \circ \beta=\beta \circ f_{\lambda}, \\
& f_{\lambda}\left(\left[a_{\mu} b\right]\right)=\left[f_{\lambda}(a)_{\lambda+\mu} \alpha^{k} \beta^{l}(b)\right]+(-1)^{|a||f|}\left[\alpha^{k} \beta^{l}(a)_{\mu} f_{\lambda}(b)\right],
\end{aligned}
$$

for all homogeneous elements $a, b \in \mathcal{R}$. 
Denote by $\operatorname{Der}_{\alpha^{k} \beta^{l}}$ the set of $\alpha^{k} \beta^{l}$-derivations of the BiHom-Lie conformal superalgebra $(\mathcal{R}, \alpha, \beta)$. For any $a \in \mathcal{R}$ satisfying $\alpha(a)=a$, $\beta(a)=a$, define $f_{k, l}: \mathcal{R} \rightarrow \mathcal{R}$ by

$$
f_{k, l}(a)_{\lambda}(b)=\left[a_{\lambda} \alpha^{k+1} \beta^{l-1}(b)\right], \quad \forall b \in \mathcal{R} .
$$

Then $f_{k, l}(a)$ is an $\alpha^{k+1} \beta^{l}$-derivation, which is called an inner $\alpha^{k+1} \beta^{l}$. derivation. In fact,

$$
\begin{aligned}
f_{k, l}(a)_{\lambda}(\partial b)= & {\left[a_{\lambda} \alpha^{k+1} \beta^{l-1}(\partial b)\right]=\left[a_{\lambda} \partial \alpha^{k+1} \beta^{l-1}(b)\right]=(\partial+\lambda) f_{k, l}(a)_{\lambda}(b) } \\
f_{k, l}(a)_{\lambda}(\alpha(b))= & {\left[a_{\lambda} \alpha^{k+2} \beta^{l-1}(b)\right]=\alpha\left[a_{\lambda} \alpha^{k+1} \beta^{l-1}(b)\right]=\alpha \circ f_{k, l}(a)_{\lambda}(b) } \\
f_{k, l}(a)_{\lambda}(\beta(b))= & {\left[a_{\lambda} \alpha^{k+1} \beta^{l}(b)\right]=\beta\left[a_{\lambda} \alpha^{k+1} \beta^{l-1}(b)\right]=\beta \circ f_{k, l}(a)_{\lambda}(b) } \\
f_{k, l}(a)_{\lambda}\left(\left[b_{\mu} c\right]\right)= & {\left[a_{\lambda} \alpha^{k+1} \beta^{l-1}\left(\left[b_{\mu} c\right]\right)=\left[\alpha \beta(a)_{\lambda}\left[\alpha^{k+1} \beta^{l-1}(b)_{\mu} \alpha^{k+1} \beta^{l-1}(c)\right]\right.\right.} \\
= & {\left.\left[\beta(a)_{\lambda} \alpha^{k+1} \beta^{l-1}(b)\right]_{\lambda+(-1)^{|a||b|} \mu} \alpha^{k+1} \beta^{l}(c)\right] } \\
& +\left[\alpha^{k+1} \beta^{l}(b)_{\mu}\left[\alpha(a)_{\lambda} \alpha^{k+1} \beta^{l-1}(c)\right]\right] \\
= & {\left.\left[a_{\lambda} \alpha^{k+1} \beta^{l-1}(b)\right]_{\lambda+\mu} \alpha^{k+1} \beta^{l}(c)\right] } \\
& +(-1)^{|a||b|}\left[\alpha^{k+1} \beta^{l}(b)_{\mu}\left[a_{\lambda} \alpha^{k+1} \beta^{l-1}(c)\right]\right] \\
= & {\left[f_{k, l}(a)_{\lambda}(b)_{\lambda+\mu} \alpha^{k+1} \beta^{l}(c)\right] } \\
& +(-1)^{|a||b|}\left[\alpha^{k+1} \beta^{l}(b)_{\mu}\left(f_{k, l}(a)_{\lambda}(c)\right)\right] .
\end{aligned}
$$

Denote by $\operatorname{Inn}_{\alpha^{k} \beta^{l}}(\mathcal{R})$ the set of inner $\alpha^{k} \beta^{l}$-derivations. For $f_{\lambda} \in \operatorname{Der}_{\alpha^{k} \beta^{l}}(\mathcal{R})$ and $g_{\mu-\lambda} \in \operatorname{Der}_{\alpha^{s} \beta^{t}}(R)$, define their commutator $\left[f_{\lambda} g\right]_{\mu}$ by

$$
\left[f_{\lambda} g\right]_{\mu}(a)=f_{\lambda}\left(g_{\mu-\lambda} a\right)-(-1)^{|f||g|} g_{\mu-\lambda}\left(f_{\lambda} a\right), \quad \forall a \in \mathcal{R}
$$


Lemma 4.1. For any $f_{\lambda} \in \operatorname{Der}_{\alpha^{k} \beta^{l}}(\mathcal{R})$ and $g_{\mu-\lambda} \in \operatorname{Der}_{\alpha^{s} \beta^{t}}(\mathcal{R})$, we have

$$
\left[f_{\lambda} g\right] \in \operatorname{Der}_{\alpha^{k+s} \beta^{l+t}}(\mathcal{R})[\lambda] .
$$

Proof. For any $a, b \in \mathcal{R}$, we have

$$
\begin{aligned}
{\left[f_{\lambda} g\right]_{\mu}(\partial a) } & =f_{\lambda}\left(g_{\mu-\lambda} \partial a\right)-(-1)^{|f||g|} g_{\mu-\lambda}\left(f_{\lambda} \partial a\right) \\
& =f_{\lambda}\left((\partial+\mu-\lambda) g_{\mu-\lambda} a\right)+(-1)^{|f||g|} g_{\mu-\lambda}\left((\mu+\lambda) f_{\lambda} a\right) \\
& =(\partial+\mu) f_{\lambda}\left(g_{\mu-\lambda} a\right)-(-1)^{|f||g|}(\partial+\mu) g_{\mu-\lambda}\left(f_{\lambda} a\right) \\
& =(\partial+\mu)\left[f_{\lambda} g\right]_{\mu}(a) .
\end{aligned}
$$

Moreover, we have

$$
\begin{aligned}
{\left[f_{\lambda} g\right]_{\mu}\left(\left[a_{\gamma} b\right]\right)=} & f_{\lambda}\left(g_{\mu-\lambda}\left[a_{\gamma} b\right]\right)-(-1)^{|f||g|} g_{\mu-\lambda}\left(f_{\lambda}\left[a_{\gamma} b\right]\right) \\
= & f_{\lambda}\left(\left[g_{\mu-\lambda}(a)_{\mu-\lambda+\gamma} \alpha^{s} \beta^{t}(b)\right]+(-1)^{|a||g|}\left[\alpha^{s} \beta^{t}(a)_{\gamma} g_{\mu-\lambda}(b)\right]\right) \\
& -(-1)^{|f||g|} g_{\mu-\lambda}\left(\left[f_{\lambda}(a)_{\lambda+\gamma} \alpha^{k} \beta^{l}(b)\right]\right. \\
& \left.+(-1)^{|f||\alpha|}\left[\alpha^{k} \beta^{l}(a)_{\gamma} f_{\lambda}(b)\right]\right) \\
= & {\left[f_{\lambda}\left(g_{\mu-\lambda}(a)\right)_{\mu+\gamma} \alpha^{k+s} \beta^{l+t}(b)\right] } \\
& +(-1)^{|f||g||a|}\left[\alpha^{k} \beta^{l}\left(g_{\mu-\lambda}(a)\right)_{\mu-\lambda+\gamma} f_{\lambda}\left(\alpha^{s} \beta^{t}(b)\right)\right] \\
& +(-1)^{|\alpha||g|}\left[f_{\lambda}\left(\alpha^{s} \beta^{t}(a)\right)_{\lambda+\gamma} \alpha^{k} \beta^{l}\left(g_{\mu-\lambda}(b)\right)\right] \\
& +(-1)^{|f||g||\alpha|}\left[\alpha^{k+s} \beta^{l+t}(a)_{\gamma}\left(f_{\lambda}\left(g_{\mu-\lambda}(b)\right)\right)\right] \\
& -(-1)^{|f||g|}\left[\left(g_{\mu-\lambda} f_{\lambda}(a)\right)_{\mu+\gamma} \alpha^{k+s} \beta^{l+t}(b)\right] \\
& -\left[\alpha^{s} \beta^{t}\left(f_{\lambda}(a)\right)_{\lambda+\gamma}\left(g_{\mu-\lambda}\left(\alpha^{k} \beta^{l}(b)\right)\right)\right]
\end{aligned}
$$




$$
\begin{aligned}
& -(-1)^{|f||g||a|}\left[\left(g_{\mu-\lambda}\left(\alpha^{k} \beta^{l}(a)\right)\right)_{\mu-\lambda+\gamma} \alpha^{s} \beta^{t}\left(f_{\lambda}(b)\right)\right] \\
& -(-1)^{|f||g||a|}\left[\alpha^{k+s} \beta^{l+t}(a)_{\lambda}\left(g_{\mu-\lambda}\left(f_{\lambda}(b)\right)\right)\right] \\
= & {\left[\left(\left[f_{\lambda} g\right]_{\mu} a\right)_{\mu+\gamma} \alpha^{k+s} \beta^{l+t}(b)\right] } \\
& +(-1)^{|f||g||a|}\left[\alpha^{k+s} \beta^{l+t}(a)_{\gamma}\left(\left[f_{\lambda} g\right]_{\mu} b\right)\right] .
\end{aligned}
$$

Therefore, $\left[f_{\lambda} g\right] \in \operatorname{Der}_{\alpha^{k+s} \beta^{l+t}}(\mathcal{R})[\lambda]$.

Define

$$
\operatorname{Der}(\mathcal{R})=\underset{k, l l \geq 0}{\bigoplus} \operatorname{Der}_{\alpha^{k} \beta^{l}}(\mathcal{R})
$$

Proposition 4.1. $\left(\operatorname{Der}(\mathcal{R}), \alpha^{\prime}, \beta^{\prime}\right)$ is a BiHom-Lie conformal superalgebra with respect to (4.2) where $\alpha^{\prime}(f)=f \circ \alpha$ and $\beta^{\prime}(f)=f \circ \beta$.

Proof. It is straightforward. So we omit details.

Let $\mathcal{R}$ be a BiHom-Lie conformal superalgebra. Define $\operatorname{Der}(\mathcal{R})_{\theta}$ is the set of all derivations of degree $\theta$, then it is obvious that $\operatorname{Der}(\mathcal{R})=$ $\operatorname{Der}(\mathcal{R})_{0} \oplus \operatorname{Der}(\mathcal{R})_{1}$ is a subalgebra of $\operatorname{Cend}(\mathcal{R})$.

Define

$$
\Omega=\left\{f_{\lambda} \in g c(\mathcal{R}) \mid f_{\lambda} \circ \alpha=\alpha \circ f_{\lambda}, f_{\lambda} \circ \beta=\beta \circ f_{\lambda}\right\} .
$$

Let $\alpha^{\prime}, \beta^{\prime}: \Omega \rightarrow \Omega, \alpha^{\prime}\left(f_{\lambda}\right)=f_{\lambda} \circ \alpha, \beta^{\prime}\left(f_{\lambda}\right)=f_{\lambda} \circ \beta$. Then $\left(\Omega, \alpha^{\prime}, \beta^{\prime}\right)$ is a BiHom-Lie conformal algebra with respect to (2.6) and $\operatorname{Der}(\mathcal{R})$ is a BiHom-conformal subalgebra of $\Omega$. 
Definition 4.2. An element $f$ in $\Omega$ is called

(a) an $\alpha^{k} \beta^{l}$-generalized derivation of $\mathcal{R}$, if there exist $f^{\prime}, f^{\prime \prime} \in \Omega$ such that $|f|=\left|f^{\prime}\right|=\left|f^{\prime \prime}\right|$ and

$$
\left[\left(f_{\lambda}(a)\right)_{\lambda+\mu} \alpha^{k} \beta^{l}(b)\right]+(-1)^{|f||a|}\left[\alpha^{k} \beta^{l}(a)_{\mu}\left(f_{\lambda}^{\prime}(b)\right)\right]=f_{\lambda}^{\prime \prime}\left(\left[a_{\mu} b\right]\right),
$$

for all homogeneous elements $a, b \in \mathcal{R}$.

(b) an $\alpha^{k} \beta^{l}$-quasiderivation of $\mathcal{R}$, if there is $f^{\prime} \in \Omega$ such that $|f|=\left|f^{\prime}\right|$ and

$$
\left[\left(f_{\lambda}(a)\right)_{\lambda+\mu} \alpha^{k} \beta^{l}(b)\right]+(-1)^{|f||a|}\left[\alpha^{k} \beta^{l}(a)_{\mu}\left(f_{\lambda}(b)\right)\right]=f_{\lambda}^{\prime}\left(\left[a_{\mu} b\right]\right),
$$

for all homogeneous elements $a, b \in \mathcal{R}$.

(c) an $\alpha^{k} \beta^{l}$-centroid of $\mathcal{R}$, if it satisfies

$$
\left[\left(f_{\lambda}(a)\right)_{\lambda+\mu} \alpha^{k} \beta^{l}(b)\right]=(-1)^{|f||a|}\left[\alpha^{k} \beta^{l}(a)_{\mu}\left(f_{\lambda}(b)\right)\right]=f_{\lambda}\left(\left[a_{\mu} b\right]\right),
$$

for all homogeneous elements $a, b \in \mathcal{R}$.

(d) an $\alpha^{k} \beta^{l}$-quasicentroid of $\mathcal{R}$, if it satisfies

$$
\left[\left(f_{\lambda}(a)\right)_{\lambda+\mu} \alpha^{k} \beta^{l}(b)\right]=(-1)^{|f||\alpha|}\left[\alpha^{k} \beta^{l}(a)_{\mu}\left(f_{\lambda}(b)\right)\right],
$$

for all homogeneous elements $a, b \in \mathcal{R}$.

(e) an $\alpha^{k} \beta^{l}$-central derivation of $\mathcal{R}$, if it satisfies

$$
\left[\left(f_{\lambda}(a)\right)_{\lambda+\mu} \alpha^{k} \beta^{l}(b)\right]=f_{\lambda}\left(\left[a_{\mu} b\right]\right)=0,
$$

for all homogeneous elements $a, b \in \mathcal{R}$. 
Denote by $\operatorname{GDer}_{\alpha^{k} \beta^{l}}(\mathcal{R}), \operatorname{QDer}_{\alpha^{k} \beta^{l}}(\mathcal{R}), C_{\alpha^{k} \beta^{l}}(\mathcal{R}), Q C_{\alpha^{k} \beta^{l}}(\mathcal{R}) \quad$ and $\operatorname{ZDer}_{\alpha^{k} \beta^{l}}(\mathcal{R})$ the sets of all $\alpha^{k} \beta^{l}$-generalized derivations, $\alpha^{k} \beta^{l}$ quasiderivations, $\alpha^{k} \beta^{l}$ centroids, $\alpha^{k} \beta^{l}$ quasicentroids and $\alpha^{k} \beta^{l}$ central derivations of $\mathcal{R}$. Set

$$
\begin{gathered}
\operatorname{GDer}(\mathcal{R}):=\bigoplus_{k \geq 0, l \geq 0} \operatorname{GDer}_{\alpha^{k} \beta^{l}}(\mathcal{R}), \operatorname{QDer}(\mathcal{R}):=\bigoplus_{k \geq 0, l \geq 0} \operatorname{QDer}_{\alpha^{k} \beta^{l}}(\mathcal{R}) . \\
C(\mathcal{R}):=\bigoplus_{k \geq 0, l \geq 0} C_{\alpha^{k} \beta^{l}}(\mathcal{R}), Q C_{\alpha^{k} \beta^{l}}(\mathcal{R}):=\bigoplus_{k \geq 0, l \geq 0} Q C_{\alpha^{k} \beta^{l}}(\mathcal{R}), \\
\operatorname{ZDer}(\mathcal{R}):=\bigoplus_{k \geq 0, l \geq 0} \operatorname{ZDer}_{\alpha^{k} \beta^{l}}(\mathcal{R}) .
\end{gathered}
$$

It is easy to see that

$$
\begin{gathered}
\mathrm{ZDer}(\mathcal{R}) \subseteq \operatorname{Der}(\mathcal{R}) \subseteq \mathrm{QDer}(\mathcal{R}) \subseteq \operatorname{GDer}(\mathcal{R}) \subseteq \operatorname{Cend}(\mathcal{R}), \\
C(\mathcal{R}) \subseteq Q C(\mathcal{R}) \subseteq \operatorname{GDer}(\mathcal{R})
\end{gathered}
$$

Proposition 4.2. Let $(\mathcal{R}, \alpha, \beta)$ be a BiHom-Lie conformal superalgebra. Then

(i) $G \operatorname{Der}(\mathcal{R}), Q \operatorname{Der}(\mathcal{R})$ and $C(\mathcal{R})$ are BiHom-Lie conformal subalgebras of $\Omega$,

(ii) $Z \operatorname{Der}(\mathcal{R})$ is a BiHom-Lie conformal ideal of $\operatorname{Der}(\mathcal{R})$.

Proof. (i) We only prove that $\operatorname{GDer}(\mathcal{R})$ is a BiHom-conformal subalgebra of $\Omega$. The proof for the other two cases can be done similarly.

For $f_{\lambda} \in \operatorname{GDer}_{\alpha^{k} \beta^{l}}(\mathcal{R}), g_{\lambda} \in \operatorname{GDer}_{\alpha^{s} \beta^{t}}(\mathcal{R}), a, b \in \mathcal{R}$, there exist $f^{\prime}, f^{\prime \prime} \in \Omega$ (resp., $g^{\prime}, g^{\prime \prime} \in \Omega$ ) such that Equation (4.4) holds for $f$ (resp., g). We only need to show

$$
\begin{aligned}
{\left[f_{\lambda}^{\prime \prime} g^{\prime \prime}\right]_{\theta}\left(\left[a_{\mu} b\right]\right)=} & {\left[\left(\left[f_{\lambda} g\right]_{\theta}(a)\right)_{\mu+\theta} \alpha^{k+s} \beta^{l+t}(b)\right] } \\
& +(-1)^{(|f|+|g|)|a|}\left[\alpha^{k+s} \beta^{l+t}(a)_{\mu}\left(\left[f_{\lambda}^{\prime} g^{\prime}\right]_{\theta}(b)\right)\right] .
\end{aligned}
$$


In fact, we have

$$
\begin{aligned}
{\left[\left(\left[f_{\lambda} g\right]_{\theta}(a)\right)_{\mu+\theta} \alpha^{k+s} \beta^{l+t}(b)\right]=} & {\left[\left(f_{\lambda}\left(g_{\theta-\lambda}(a)\right)\right)_{\mu+\theta} \alpha^{k+s} \beta^{l+t}(b)\right] } \\
& -(-1)^{|f||g|}\left[\left(g_{\theta-\lambda}\left(f_{\lambda}(a)\right)\right)_{\mu+\theta} \alpha^{k+s} \beta^{l+t}(b)\right] .
\end{aligned}
$$

On the other hand,

$$
\begin{aligned}
& {\left[\left(f_{\lambda}\left(g_{\theta-\lambda}(a)\right)\right)_{\mu+\theta} \alpha^{k+s} \beta^{l+t}(b)\right] } \\
&= f_{\lambda}^{\prime \prime}\left(\left[\left(g_{\theta-\lambda}(a)\right)_{\mu+\theta-\lambda} \alpha^{s} \beta^{t}(b)\right]\right)-(-1)^{|f|(|g|+|a|)} \\
& \times\left[\alpha^{k} \beta^{l}\left(g_{\theta-\lambda}(a)\right)_{\mu+\theta-\lambda}\left(f_{\lambda}^{\prime}\left(\alpha^{s} \beta^{t}(b)\right)\right)\right] \\
&= f_{\lambda}^{\prime \prime}\left(g_{\theta-\lambda}^{\prime \prime}\left(\left[a_{\mu} b\right]\right)\right)-(-1)^{|g||a|} f_{\lambda}^{\prime \prime}\left(\left[\alpha^{s} \beta^{t}(a)_{\mu}\left(g_{\theta-\lambda}^{\prime}(b)\right)\right]\right) \\
&-(-1)^{|f|(|g|+|a|)} g_{\theta-\lambda}^{\prime \prime}\left(\left[\alpha^{s} \beta^{t}(a)_{\mu}\left(f_{\lambda}^{\prime}(b)\right)\right]\right) \\
&+(-1)^{|f|(|g|+|a|)+|g||a|}\left[\alpha^{k+s} \beta^{l+t}(a)_{\mu}\left(g_{\theta-\lambda}^{\prime}\left(f_{\lambda}^{\prime}(b)\right)\right)\right],
\end{aligned}
$$

and

$$
\begin{aligned}
& {\left[\left(g_{\theta-\lambda}\left(f_{\lambda}(a)\right)\right)_{\mu+\theta} \alpha^{k+s} \beta^{l+t}(b)\right] } \\
&= g_{\theta-\lambda}^{\prime \prime}\left(\left[\left(f_{\lambda}(a)\right)_{\lambda+\mu} \alpha^{k} \beta^{l}(b)\right]\right)-(-1)^{|g|} \mid(f|+| a \mid) \\
& \times\left[f_{\lambda}\left(\alpha^{s} \beta^{t}(a)\right)_{\lambda+\mu}\left(g_{\theta-\lambda}^{\prime}\left(\alpha^{k} \beta^{l}(b)\right)\right)\right] \\
&= g_{\theta-\lambda}^{\prime \prime}\left(f_{\lambda}^{\prime \prime}\left(\left[a_{\mu} b\right]\right)\right)-(-1)^{|f||a|} g_{\theta-\lambda}^{\prime \prime}\left(\left[\alpha^{k} \beta^{l}(a)_{\mu}\left(f_{\lambda}^{\prime}(b)\right)\right]\right) \\
&-(-1)^{|g|(|f|+|a|)} f_{\lambda}^{\prime \prime}\left(\left[\alpha^{s} \beta^{t}(a)_{\mu}\left(g_{\theta-\lambda}^{\prime}(b)\right)\right]\right) \\
&+(-1)^{|g|(|f|+|a|)+|f||a|}\left[\alpha^{k+s} \beta^{l+t}(a)_{\mu} f_{\lambda}^{\prime}\left(g_{\theta-\lambda}^{\prime}(b)\right)\right] .
\end{aligned}
$$


Substituting Equations (4.12) and (4.13) into Equation (4.11), we obtain Equation (4.10). In addition, It is obvious that $\alpha^{\prime}(f) \in \operatorname{GDer}(\mathcal{R})$ and $\beta^{\prime}(f) \in \operatorname{GDer}(\mathcal{R})$. Hence $\left[f_{\lambda} g\right] \in \operatorname{GDer}(\mathcal{R})[\lambda]$, and $\operatorname{GDer}(\mathcal{R})$ is a BiHom-conformal subalgebra of $\Omega$.

(ii) For $f \in \operatorname{ZDer}_{\alpha^{k} \beta^{l}}(\mathcal{R}), g \in \operatorname{Der}_{\alpha^{s} \beta^{t}}(\mathcal{R})$, and $a, b \in \mathcal{R}$, we have

$$
\begin{aligned}
{\left[f_{\lambda} g\right]_{\theta}\left(\left[a_{\mu} b\right]\right)=} & f_{\lambda}\left(g_{\theta-\lambda}\left(\left[a_{\mu} b\right]\right)\right)-(-1)^{|f||g|} g_{\theta-\lambda}\left(f_{\lambda}\left(\left[a_{\mu} b\right]\right)\right) \\
= & f_{\lambda}\left(g_{\theta-\lambda}\left(\left[a_{\mu} b\right]\right)\right)=f_{\lambda}\left(\left[\left(g_{\theta-\lambda}(a)\right)_{\mu+\theta-\lambda} \alpha^{s} \beta^{t}(b)\right]\right. \\
& \left.+(-1)^{|f||g|}\left[\alpha^{s} \beta^{t}(a)_{\mu}\left(g_{\theta-\lambda}(b)\right)\right]\right)=0,
\end{aligned}
$$

and

$$
\begin{aligned}
{\left[\left[f_{\lambda} g\right]_{\theta}(a)_{\mu+\theta} \alpha^{k+s} \beta^{l+t}(b)\right]=} & {\left[\left(f_{\lambda}\left(g_{\theta-\lambda}(a)\right)-(-1)^{|f||g|} g_{\theta-\lambda}\left(f_{\lambda}(a)\right)\right)_{\mu+\theta} \alpha^{k+s} \beta^{l+t}(b)\right] } \\
= & {\left[-(-1)^{|f||g|}\left(g_{\theta-\lambda}\left(f_{\lambda} a\right)\right)_{\mu+\theta} \alpha^{k+s} \beta^{l+t}(b)\right] } \\
= & -(-1)^{|f||g|} g_{\theta-\lambda}^{\prime \prime}\left(\left[f_{\lambda}(a)_{\lambda+\mu} \alpha^{k} \beta^{l+t}(b)\right]\right) \\
& +(-1)^{|g||a|}\left[f_{\lambda}\left(\alpha^{s} \beta^{t}(a)\right)_{\lambda+\mu} g_{\theta-\lambda}^{\prime}\left(\alpha^{k} \beta^{t}(b)\right)\right] \\
= & 0 .
\end{aligned}
$$

This means that $\left[f_{\lambda} g\right] \in \operatorname{ZDer}(\mathcal{R})[\lambda]$. Thus $\operatorname{ZDer}(\mathcal{R})$ is an ideal of $\operatorname{Der}(\mathcal{R})$.

Proposition 4.3. Let $f \in Q C_{\alpha^{k} \beta^{l}}(\mathcal{R})$ and $g \in Q C_{\alpha^{s} \beta^{l}}(\mathcal{R})$. Then $\left[f_{\lambda} g\right]$ is an $\alpha^{k+s} \beta^{l+t}$-generalized derivation of degree $|f|+|g|$. 
Proof. Assume that $f \in Q C_{\alpha^{k} \beta^{l}}(\mathcal{R})$ and $g \in Q C_{\alpha^{s} \beta^{l}}(\mathcal{R})$. Then for any homogeneous elements $a, b \in \mathcal{R}$, we have

$$
\left[\left(f_{\lambda}(a)\right)_{\lambda+\mu} \alpha^{k} \beta^{l}(b)\right]=(-1)^{|f||a|}\left[\alpha^{k} \beta^{l}(a)_{\mu}\left(f_{\lambda}(b)\right)\right],
$$

and

$$
\left[\left(g_{\lambda}(a)\right)_{\lambda+\mu} \alpha^{s} \beta^{t}(b)\right]=(-1)^{|g||\alpha|}\left[\alpha^{s} \beta^{t}(a)_{\mu}\left(g_{\lambda}(b)\right)\right] .
$$

Hence

$$
\begin{aligned}
{\left[\left[f_{\lambda} g\right]_{\theta}(a)_{\mu+\theta} \alpha^{k+s} \beta^{l+t}(b)\right]=} & {\left[f_{\lambda}\left(g_{\theta-\lambda}(a)\right)_{\mu+\theta} \alpha^{k+s} \beta^{l+t}(b)\right] } \\
& -(-1)^{|f||g|}\left[g_{\theta-\lambda}\left(f_{\lambda}(a)\right)_{\mu+\theta} \alpha^{k+s} \beta^{l+t}(b)\right] \\
& =(-1)^{(|f|+|g|)|a|}\left[\alpha^{k+s} \beta^{l+t}(a)_{\mu} g_{\theta-\lambda}\left(f_{\lambda}(b)\right)\right] \\
& -(-1)^{(|f|+|g|)|a|}(-1)^{|f||g|}\left[\alpha^{k+s} \beta^{l+t}(a)_{\mu} f_{\lambda}\left(g_{\theta-\lambda}(b)\right)\right] \\
& =-(-1)^{(|f|+|g|)|\alpha|}\left[\alpha^{k+s} \beta^{l+t}(a)_{\mu}(-1)^{|f||g|}\left[f_{\lambda} g\right]_{\theta}(b)\right],
\end{aligned}
$$

which implies that

$\left[\left[f_{\lambda} g\right]_{\theta}(a)_{\mu+\theta} \alpha^{k+s} \beta^{l+t}(b)\right]+(-1)^{(|f|+|g|)|\alpha|}\left[\alpha^{k+s} \beta^{l+t}(a)_{\mu}(-1)^{|f||g|}\left[f_{\lambda} g\right]_{\theta}(b)\right]=0$.

Then $\left[f_{\lambda} g\right]$ is an $\alpha^{k+s} \beta^{l+t}$-generalized derivation of degree $|f|+|g|$.

Proposition 4.4. Let $(\mathcal{R}, \alpha, \beta)$ be a BiHom-Lie conformal superalgebra. If $f \in Q D e r_{\alpha^{k} \beta^{l}}(\mathcal{R})$ and $g \in Q C_{\alpha^{k} \beta^{t}}(\mathcal{R})$ such that $|f|=|g|$, then $f+g \in G \operatorname{Der}_{\alpha^{k} \beta^{l}}(\mathcal{R})$ with degree $|f|$.

Proof. Let $f_{\lambda} \in \operatorname{QDer}_{\alpha^{k} \beta^{l}}(\mathcal{R})$. Then there exist $f^{\prime} \in \Omega$ such that

$$
\left[\left(f_{\lambda}(a)\right)_{\lambda+\mu} \alpha^{k} \beta^{l}(b)\right]+(-1)^{|f||a|}\left[\alpha^{k} \beta^{l}(a)_{\mu}\left(f_{\lambda}(b)\right)\right]=f_{\lambda}^{\prime}\left(\left[a_{\mu} b\right]\right), \forall a, b \in \mathcal{R}
$$


And let $g \in Q C_{\alpha^{k} \beta^{t}}(\mathcal{R})$. Then

$$
\left[\left(g_{\lambda}(a)\right)_{\lambda+\mu} \alpha^{k} \beta^{l}(b)\right]=(-1)^{|f||\alpha|}\left[\alpha^{k} \beta^{l}(a)_{\mu}\left(g_{\lambda}(b)\right)\right], \forall a, b \in \mathcal{R}
$$

We will prove that $f+g$ satisfies Equation (4.4). In fact,

$$
\begin{aligned}
{\left[(f+g)_{\lambda}(a)_{\lambda+\mu} \alpha^{k} \beta^{l}(b)\right]=} & {\left[f_{\lambda}(a)_{\lambda+\mu} \alpha^{k} \beta^{l}(b)\right]+\left[g_{\lambda}(a)_{\lambda+\mu} \alpha^{k} \beta^{l}(b)\right] } \\
= & f_{\lambda}^{\prime}\left(\left[a_{\mu} b\right]\right)-(-1)^{|f||\alpha|}\left[\alpha^{k} \beta^{l}(a)_{\mu} f_{\lambda}(b)\right] \\
& +(-1)^{|f||a|}\left[\alpha^{k} \beta^{l}(a)_{\mu} g_{\lambda}(b)\right] \\
= & f_{\lambda}^{\prime}\left(\left[a_{\mu} b\right]\right)-(-1)^{|f||\alpha|}\left[\alpha^{k} \beta^{l}(a)_{\mu}(f-g)_{\lambda}(b)\right] .
\end{aligned}
$$

Then

$$
\left[(f+g)_{\lambda}(a)_{\lambda+\mu} \alpha^{k} \beta^{l}(b)\right]+(-1)^{|f||a|}\left[\alpha^{k} \beta^{l}(a)_{\mu}(f-g)_{\lambda}(b)\right]=f_{\lambda}^{\prime}\left(\left[a_{\mu} b\right]\right) .
$$

Therefore $f+g$ satisfies Equation (4.4). This completes the proof.

\section{References}

[1] F. Ammar and A. Makhlouf, Hom-Lie superalgebras and Hom-Lie admissible superalgebras, Journal of Algebra 324(7) (2010), 1513-1528.

DOI: https://doi.org/10.1016/j.jalgebra.2010.06.014

[2] F. Ammar, A. Makhlouf and N. Saadaoui, Cohomology of Hom-Lie superalgebras and $q$-deformed Witt superalgebra, Czechoslovak Mathematical Journal 63(3) (2013), 721-761.

DOI: https://doi.org/10.1007/s10587-013-0049-6

[3] F. Davide, V. G. Kac and A. Retakh, Structure Theory of Finite Lie Conformal Superalgebras, Lie Theory and its Applications in Physics V (2004), 27-63.

DOI: https://doi.org/10.1142/9789812702562_0002

[4] G. Fan, Y. Hong and Y. Su, Generalized conformal derivations of Lie conformal algebras, Journal of Algebra and its Applications 18(9) (2019); Article 1950175.

DOI: https://doi.org/10.1142/S0219498819501755 
[5] G. Graziani, A. Makhlouf, C. Menini and F. Panaite, BiHom-associative algebras, BiHom-Lie algebras and BiHom-bialgebras, Symmetry, Integrability and Geometry, Methods and Applications 11 (2015); Article 86, 1-34.

DOI: https://doi.org/10.3842/SIGMA.2015.086

[6] I. M. Gel'fand and I. Ya. Dorfman, Hamiltonian operators and algebraic structures related to them, Functional Analysis and its Applications 13(4) (1979), 13-30.

DOI: https://doi.org/10.1007/BF01078363

[7] S. Guo and S. Wang, On $\partial$-Hom-Jordan Lie conformal superalgebras, Journal of Geometry and Physics 155 (2020); Article 103745.

DOI: https://doi.org/10.1016/j.geomphys.2020.103745

[8] S. Guo, L. Dong and S. Wang, Representations and derivations of Hom-Lie conformal superalgebras, arXiv preprint arXiv:1807.03638 (2018).

[9] S. Guo, X. Zhang and S. Wang, Cohomology and derivations of BiHom-Lie conformal algebras, arXiv preprint arXiv:1808.09811 (2018).

[10] V. G. Kac, Vertex Algebras for Beginners, University Lecture Series 10, (American Mathematical Society), Providence, RI, 1996, Second Edition, 1998.

[11] X. Xu, Quadratic conformal superalgebras, Journal of Algebra 231(1) (2000), 1-38.

DOI: https://doi.org/10.1006/jabr.1999.8346

[12] L. Yuan, Hom Gel'fand-Dorfman bialgebras and Hom-Lie conformal algebras, Journal of Mathematical Physics 55(4) (2014); Article 043507.

DOI: https://doi.org/10.1063/1.4870870

[13] L. Yuan, S. Chen and C. He, Hom-Gel'fand-Dorfman super-bialgebras and Hom-Lie conformal superalgebras, Acta Mathematica Sinica: English Series 33(1) (2017), 96-116.

DOI: https://doi.org/10.1007/s10114-016-6074-2

[14] J. Zhao, L. Chen and L. Yuan, Deformations and generalized derivations of Lie conformal superalgebras, Journal of Mathematical Physics 58(11) (2017); Article 111702

DOI: https://doi.org/10.1063/1.5012886

[15] J. Zhao, L. Yuan and L. Chen, Deformations and generalized derivations of Hom-Lie conformal algebras, Science China Mathematics 61(5) (2018), 797-812.

DOI: https://doi.org/10.1007/s11425-016-9063-0 